\title{
A METHOD FOR DETERMINING THE PHYSICAL PROPERTIES OF THE COLDEST KNOWN BROWN DWARFS
}

\author{
AdAm J. Burgasser ${ }^{1}$ \\ Massachusetts Institute of Technology, Kavli Institute for Astrophysics and Space Research, 77 Massachusetts Avenue, \\ Building 37, Cambridge, MA 02139-4307; ajb@mit.edu \\ AdAm Burrows \\ Department of Astronomy, University of Arizona, Tucson, AZ 85721; burrows@as.arizona.edu \\ AND \\ J. DAVY KIRKPATRICK \\ Infrared Processing and Analysis Center, Mail Stop 100-22, California Institute of Technology, Pasadena, \\ CA 91125; davy@ipac.caltech.edu \\ Received 2005 March 4; accepted 2005 October 20
}

\begin{abstract}
We present a method for measuring the physical parameters of the coldest T-type brown dwarfs using lowresolution near-infrared spectra. By comparing $\mathrm{H}_{2} \mathrm{O}$ and $\mathrm{H}_{2}$-sensitive spectral ratios between empirical data and theoretical atmosphere models, and calibrating these ratios to measurements for the well-characterized 2-5 Gyr companion brown dwarf Gliese 570D, we derive estimates of the effective temperatures and surface gravities for 13 mid- and late-type field $\mathrm{T}$ dwarfs. We also deduce the first quantitative estimate of subsolar metallicity for the peculiar T dwarf 2MASS 0937+2931. Derived temperatures are consistent with prior estimates based on parallax and bolometric luminosity measurements, and examination of possible systematic effects indicate that the results are robust. Two recently discovered late-type T dwarfs, 2MASS 0939-2448 and 2MASS 1114-2618, both appear to be $\gtrsim 50 \mathrm{~K}$ cooler than the latest type T dwarf, 2MASS 0415-0935, and are potentially the coldest and least luminous brown dwarfs currently known. We find that, in general, higher surface gravity $\mathrm{T}$ dwarfs have lower effective temperatures and luminosities for a given spectral type, explaining previously observed scatter in the $T_{\text {eff }} /$ spectral type relation for these objects. Masses, radii, and ages are estimated for the $\mathrm{T}$ dwarfs in our sample using the evolutionary models of Burrows et al.; we also determine masses and radii independently for eight $\mathrm{T}$ dwarfs with measured luminosities. These two determinations are largely consistent, lending support to the validity of evolutionary models at late ages. Our method is well suited to large samples of faint brown dwarfs and can ultimately be used to directly measure the substellar mass function and formation history in the Galaxy.
\end{abstract}

Subject headings: stars: fundamental parameters stars: individual (2MASS J09373487+2931409, 2MASS J09393548-2448279, 2MASS J11145133-2618235, Gliese 570D) - stars: low-mass, brown dwarfs

Online material: color figures

\section{INTRODUCTION}

The spectral energy distributions of the coldest known stars and brown dwarfs, L dwarfs and T dwarfs (Kirkpatrick et al. 1999; Burgasser et al. 2002b; Geballe et al. 2002), are complex and dominated by broad, overlapping gaseous and condensate molecular absorption features. The strengths of these features depend on a combination of photospheric temperature, gas pressure, and composition (e.g., Burrows \& Sharp 1999; Lodders $\&$ Fegley 2002), which in turn are related to the effective temperature $\left(T_{\text {eff }}\right)$, surface gravity $(g)$, and metallicity $([\mathrm{M} / \mathrm{H}])$ of a brown dwarf. Nonequilibrium effects (e.g., vertical mixing, cloud coverage) may also play an important role in molecular (Fegley \& Lodders 1996; Saumon et al. 2003) and condensate (Ackerman $\&$ Marley 2001; Burgasser et al. 2002a) abundances. The combined influence of these parameters on the spectra of $\mathrm{L}$ and

\footnotetext{
${ }^{1}$ Visiting Astronomer at the Infrared Telescope Facility, which is operated by the University of Hawaii under Cooperative Agreement NCC 5-538 with the National Aeronautics and Space Administration, Office of Space Science, Planetary Astronomy Program.
}

$\mathrm{T}$ dwarfs is only beginning to be explored through the study of low-mass, substellar objects in young clusters and stellar associations (Lucas et al. 2001; Gorlova et al. 2003; McGovern et al. 2004) and ultracool subdwarfs (Burgasser et al. 2003d; Scholz et al. 2004), although systematic studies have yet to be achieved.

Disentangling the properties of $T_{\text {eff }}$, surface gravity, and metallicity is a principal goal of substellar astrophysics. These parameters can be used to infer masses, radii, and ages for individual sources (e.g., Mohanty et al. 2004b), allowing, in the long term, direct measurement of the substellar mass function (MF) and star formation history for field objects in the solar neighborhood (Chabrier 2003; Burgasser 2004; Allen et al. 2005). In the short term, $T_{\text {eff }}, g$, and $[\mathrm{M} / \mathrm{H}]$ measurements for young cluster or companion brown dwarfs enable tests of evolutionary models (Mohanty et al. 2004a).

Gravity and metallicity effects are particularly relevant for interpreting the spectral energy distributions of the coldest $\mathrm{T}$ dwarfs: spectral types T6 and later. These objects, with $T_{\text {eff }} \lesssim 1000 \mathrm{~K}$ (Golimowski et al. 2004), lack the complicating influence of photospheric condensates common in late-type M dwarfs, L dwarfs, and the earliest type T dwarfs (Tsuji et al. 1996; Ackerman \& 
Marley 2001; Allard et al. 2001). They exhibit good correlation between spectral type and $T_{\text {eff }}$ (Dahn et al. 2002; Tinney et al. 2003; Golimowski et al. 2004; Nakajima et al. 2004; Vrba et al. 2004). Surface gravity and metallicity effects are therefore readily distinguished by the presence of spectral or photometric anomalies. One case in point is the peculiar T6 dwarf 2MASS 0937+ $2931^{2}$ (Burgasser et al. 2002b), a brown dwarf believed to have a high surface gravity and/or subsolar metallicity (Burgasser et al. 2002b, 2003a; Burrows et al. 2002; Knapp et al. 2004). 2MASS $0937+2931$ is $0.5-1.0$ mag bluer than similarly classified T dwarfs, and its spectrum exhibits a suppressed $K$-band peak, an extremely red $0.8-1.0 \mu \mathrm{m}$ spectral slope, enhanced FeH absorption at $0.99 \mu \mathrm{m}$, and an absence of $\mathrm{K}$ I doublet lines at 1.17 and $1.25 \mu \mathrm{m}$, all unusual for a mid-type T dwarf. Several other late-type T dwarfs exhibit similar color and spectral peculiarities (Burgasser et al. 2003c, 2004b; Knapp et al. 2004). However, quantitative analysis of these effects, in the form of specific surface gravity and metallicity measurements, has been limited (Burrows et al. 2002; Knapp et al. 2004).

In this article, we present a method for disentangling $T_{\text {eff }}, g$, and $[\mathrm{M} / \mathrm{H}]$ effects in the near-infrared spectra of the latest type $\mathrm{T}$ dwarfs. Our method, based on the comparison of calibrated nearinfrared flux ratios measured on low-resolution spectral data and theoretical models, yields strong constraints on these physical parameters and a means of estimating masses, radii, and ages for individual field brown dwarfs. In $\S 2$ we describe the sample and spectroscopic observations obtained with the SpeX spectrograph (Rayner et al. 2003) mounted on the $3 \mathrm{~m}$ NASA Infrared Telescope Facility (IRTF). We identify and compare spectral variations observed in these low-resolution near-infrared spectra and discuss qualitatively how these features are associated with differences in $T_{\text {eff }}, g$, and [M/H]. In $\S 3$ we examine these same effects with theoretical models and characterize spectral trends. In $\S 4$ we describe our method and present $T_{\text {eff }}$ and $\log g$ estimates for 13 field brown dwarfs and constraints for two others; we also deduce subsolar metallicity estimates for two sources, including 2MASS $0937+2931$. We demonstrate the consistency of our $T_{\text {eff }}$ values with previous determinations based on parallax and luminosity measurements and examine potential systematic effects. In $\S 5$ we derive mass, radius, and age estimates for our $\mathrm{T}$ dwarfs using the evolutionary models of Burrows et al. (1997) and independently determine masses and radii for eight sources with published luminosity measurements. We discuss the results in $\S 6$, focusing on new insights on the $T_{\text {eff }} /$ spectral type relation for $T$ dwarfs and potential applications of our method for various brown dwarf studies. Results are summarized in $\S 7$.

\section{OBSERVATIONS}

\subsection{The Sample}

Our primary spectral sample was composed of $16 \mathrm{~T}$ dwarfs identified by Strauss et al. (1999), Burgasser et al. (1999, 2002b, 2003c, 2004b), Tsvetanov et al. (2000), Geballe et al. (2002), Knapp et al. (2004), and Tinney et al. (2005) in the Two Micron All Sky Survey (2MASS; Cutri et al. 2003) and the Sloan Digital Sky Survey (SDSS; York et al. 2000). The empirical properties of these sources are listed in Table 1. The sample was selected to span types T5.5 to T8, based on the unified classification scheme of Burgasser et al. (2006), and excludes known binaries

\footnotetext{
2 Source designations in this article are abbreviated in the manner 2MASS $\mathrm{hhmm} \pm \mathrm{ddmm}$; the suffix is the sexagesimal right ascension (hours and minutes) and declination (degrees and arcminutes) at J2000.0 equinox. Full designations are provided in Table 1 .
}

(Burgasser et al. 2003b; A. J. Burgasser et al. 2006, in preparation). Eight of these objects have parallax measurements from Dahn et al. (2002), Tinney et al. (2003), and Vrba et al. (2004); all but one have a reported proper motion. Apparent 2MASS $J$-band magnitudes for these sources range from 14.7 to $16.3 \mathrm{mag}$.

\subsection{Near-Infrared Spectroscopy}

Six of the T dwarfs in our sample-2MASS 0034+0523, 2MASS 0243-2453, 2MASS 0415-0935, 2MASS 1231+0847, Gliese 570D, and 2MASS 2228-4310 - have been previously observed with SpeX (Burgasser et al. 2004b). The remaining sources were observed during three runs: 2004 March 11-12, 2004 July 23, and 2004 September 7 (UT). A log of observations is provided in Table 2. Conditions during the March run were clear and dry, with typical seeing of 0.77 . Conditions during July were also clear, with excellent seeing $\left(0{ }^{\prime \prime} 4-00^{\prime \prime} 7\right)$. Light cirrus was present during the September observations, but seeing was again excellent $\left(00^{\prime \prime} 5-00^{\prime \prime} 7\right)$.

Spectral data for all of the sources in our sample (including those previously observed) were obtained using the SpeX prism dispersed mode, which provides low-resolution $0.7-2.5 \mu \mathrm{m}$ spectra in a single order. This setting minimizes spectral color errors commonly incurred through order stitching (e.g., McLean et al. 2003), yielding an accurate measure of the broadband spectral energy distribution. For all observations, the 0.5 slit was employed and rotated to the parallactic angle, resulting in a spectral resolution $\lambda / \Delta \lambda \approx 150$ and dispersion across the chip of 20-30 $\AA$ pixel ${ }^{-1}$. Multiple exposures of $180 \mathrm{~s}$ were obtained in an ABBA dither pattern along the slit. Flux calibration was made through observations of nearby A0 $\mathrm{V}$ stars obtained immediately before or after the target observation and at similar air masses $(\Delta \sec z<$ 0.1 ). Internal flat-field and Ar arc lamps were observed after each flux calibrator star for pixel response and wavelength calibration. All spectral data were reduced using the SpeXtool package version 3.2 (Vacca et al. 2003; Cushing et al. 2004) using standard settings. Further details on the experimental design and data reduction are given in Burgasser et al. (2004b).

The reduced spectra of the newly observed $\mathrm{T}$ dwarfs are shown in Figure 1. Readily apparent are the deep molecular bands of $\mathrm{H}_{2} \mathrm{O}$ and $\mathrm{CH}_{4}$ that shape the $1.05 \mu \mathrm{m}$ ( $\mathrm{Y}$ band), $1.27 \mu \mathrm{m}$ ( $J$ band), $1.6 \mu \mathrm{m}$ ( $H$ band), and $2.1 \mu \mathrm{m}$ ( $K$ band) flux peaks, the defining features of $\mathrm{T}$ dwarf near-infrared spectra. The spectra are also shaped by the pressure-broadened red wings of the $0.77 \mu \mathrm{m} \mathrm{K} \mathrm{I}$ doublet shortward of $1 \mu \mathrm{m}$ and collision-induced $\mathrm{H}_{2}$ absorption at $K$ band, both of which are discussed in detail below. Finer atomic line features, including the 1.17 and $1.25 \mu \mathrm{m} \mathrm{K}$ I doublets, are unresolved in these data. Further discussion on the spectral characteristics of T dwarfs can be found in Burgasser et al. (2002b, 2003a), Geballe et al. (2002), McLean et al. (2003), Knapp et al. (2004), Nakajima et al. (2004), Cushing et al. (2005), and Kirkpatrick (2005).

\subsection{Spectral Signatures of Surface Gravity and Metallicity}

Variations in the near-infrared spectral features of $\mathrm{T}$ dwarfs are generally synchronized with spectral type; later subtypes exhibit both stronger $\mathrm{H}_{2} \mathrm{O}$ and $\mathrm{CH}_{4}$ bands and bluer near-infrared colors. However, slight deviations to these trends exist and are apparent when one compares sources with similar spectral types, as in Figure 2. Displayed in the left panel of this figure are the normalized spectra of three T6/T6.5 dwarfs-2MASS 0937+2931, SDSS 1346-0031, and 2MASS 2228-4310 - overlaid on that of the T6 spectral standard SDSS 1624+0029 (Burgasser et al. 2006). While $\mathrm{H}_{2} \mathrm{O}$ and $\mathrm{CH}_{4}$ bands are similar among these spectra, clear 
TABLE 1

T Dwarf SAMple

\begin{tabular}{|c|c|c|c|c|c|c|c|c|c|}
\hline \multirow[b]{2}{*}{$\begin{array}{l}\text { NAME } \\
(1)\end{array}$} & \multirow[b]{2}{*}{$\begin{array}{c}\text { SPECTRAL TyPE } \\
\text { (2) }\end{array}$} & \multicolumn{2}{|c|}{$\mathrm{J}^{2} 000.0$ COORDINATES $^{\mathrm{a}}$} & \multicolumn{3}{|c|}{ 2MASS РноTOMETRY } & \multirow{2}{*}{$\begin{array}{c}\pi \\
\text { (mas) } \\
(8)\end{array}$} & \multirow{2}{*}{$\begin{array}{c}\mu \\
\left(\operatorname{arcsec} \mathrm{yr}^{-1}\right) \\
(9)\end{array}$} & \multirow[b]{2}{*}{$\begin{array}{c}\text { REFERENCES }^{\mathrm{b}} \\
\text { (10) }\end{array}$} \\
\hline & & $\begin{array}{c}\alpha \\
(3)\end{array}$ & $\begin{array}{c}\delta \\
(4)\end{array}$ & $\begin{array}{c}J \\
(5)\end{array}$ & $\begin{array}{l}H \\
(6)\end{array}$ & $\begin{array}{l}K_{s} \\
\text { (7) }\end{array}$ & & & \\
\hline 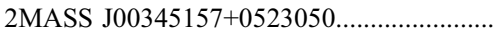 & T6.5 & 003451.57 & +052305.0 & $15.54 \pm 0.05$ & $15.44 \pm 0.08$ & $>16.2$ & $\ldots$ & $0.68 \pm 0.06$ & 1 \\
\hline 2MASS J00501994-3322402 …….............. & $\mathrm{T} 7$ & 005019.94 & -332240.2 & $15.93 \pm 0.07$ & $15.84 \pm 0.19$ & $15.24 \pm 0.19$ & $\ldots$ & $1.5 \pm 0.1$ & 2 \\
\hline 2MASS J02431371-2453298_....................... & T6 & 024313.71 & -245329.8 & $15.38 \pm 0.05$ & $15.14 \pm 0.11$ & $15.22 \pm 0.17$ & $94 \pm 4$ & $0.355 \pm 0.004$ & 3,4 \\
\hline 2MASS J04151954-0935066 …………...... & T8 & 041519.54 & -093506.6 & $15.70 \pm 0.06$ & $15.54 \pm 0.11$ & $15.43 \pm 0.20$ & $174 \pm 3$ & $2.255 \pm 0.003$ & 3,4 \\
\hline 2MASS J07271824+1710012........................ & $\mathrm{T} 7$ & 072718.24 & +17 1001.2 & $15.60 \pm 0.06$ & $15.76 \pm 0.17$ & $15.56 \pm 0.19$ & $110 \pm 2$ & $1.297 \pm 0.005$ & 3,4 \\
\hline 2MASS J09373487+2931409.......................... & T6p & 093734.87 & +293140.9 & $14.65 \pm 0.04$ & $14.70 \pm 0.07$ & $15.27 \pm 0.13$ & $163 \pm 4$ & $1.622 \pm 0.007$ & 3,4 \\
\hline 2MASS J09393548-2448279........................ & $\mathrm{T} 8$ & 093935.48 & -244827.9 & $15.98 \pm 0.11$ & $15.80 \pm 0.15$ & $>16.6$ & $\ldots$ & $1.15 \pm 0.06$ & 2 \\
\hline SDSS J111010.01+011613.1 …....................... & T5.5 & 111010.01 & +011613.0 & $16.34 \pm 0.12$ & $15.92 \pm 0.14$ & $>15.1$ & $\ldots$ & $0.34 \pm 0.10$ & 2,5 \\
\hline 2MASS J11145133-2618235 …...................... & $\mathrm{T} 7.5$ & 111451.33 & -261823.5 & $15.86 \pm 0.08$ & $15.73 \pm 0.12$ & $>16.1$ & $\ldots$ & $3.05 \pm 0.04$ & 2 \\
\hline 2MASS J1217110-0311131 ……………....... & $\mathrm{T} 7.5$ & 121711.10 & -031113.1 & $15.86 \pm 0.06$ & $15.75 \pm 0.12$ & $>15.9$ & $91 \pm 2$ & $1.0571 \pm 0.0017$ & 6,7 \\
\hline 2MASS J12314753+0847331......................... & T5.5 & 123147.53 & +084733.1 & $15.57 \pm 0.07$ & $15.31 \pm 0.11$ & $15.22 \pm 0.20$ & $\ldots$ & $1.61 \pm 0.07$ & $1,2,8$ \\
\hline SDSS J134646.45-003150.4 ...................... & T6.5 & 134646.34 & -003150.1 & $16.00 \pm 0.10$ & $15.46 \pm 0.12$ & $15.77 \pm 0.27$ & $68 \pm 2$ & $0.516 \pm 0.003$ & 7,9 \\
\hline Gliese 570D & T7.5 & 145714.96 & -212147.7 & $15.32 \pm 0.05$ & $15.27 \pm 0.09$ & $15.24 \pm 0.16$ & $169.3 \pm 1.7$ & $2.012 \pm 0.004$ & 10,11 \\
\hline SDSS J162414.37+002915.6 _......................... & T6 & 162414.36 & +00 2915.8 & $15.49 \pm 0.05$ & $15.52 \pm 0.10$ & $>15.5$ & $92 \pm 2$ & $0.3832 \pm 0.0019$ & 12,13 \\
\hline SDSS J175805.46+463311.9 ……................... & T6.5 & 175805.45 & +463309.9 & $16.15 \pm 0.09$ & $16.25 \pm 0.22$ & $15.47 \pm 0.19$ & $\ldots$ & $\ldots$ & 8 \\
\hline 2MASS J22282889-4310262 …………...... & $\mathrm{T} 6$ & 222828.89 & $-43 \quad 1026.2$ & $15.66 \pm 0.07$ & $15.36 \pm 0.12$ & $15.30 \pm 0.21$ & $\ldots$ & $0.31 \pm 0.03$ & 14 \\
\hline
\end{tabular}

NoтE.-Units of right ascension are hours, minutes, and seconds, and units of declination are degrees, arcminutes, and arcseconds.

${ }^{a}$ Right ascension $(\alpha)$ and declination $(\delta)$ at equinox J2000.0 from the 2MASS All-Sky Data Release Point-Source Catalog (Cutri et al. 2003)

Discovery, parallax, and proper motion references.

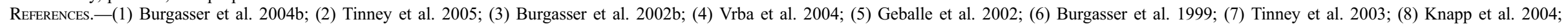
(9) Tsvetanov et al. 2000; (10) Burgasser et al. 2000; (11) Hipparcos (ESA 1997); (12) Strauss et al. 1999; (13) Dahn et al. 2002; (14) Burgasser et al. 2003c. 
TABLE 2

Log of New SpeX Observations

\begin{tabular}{|c|c|c|c|c|c|}
\hline $\begin{array}{l}\text { Source } \\
\text { (1) }\end{array}$ & $\begin{array}{l}\text { UT Date } \\
\text { (2) }\end{array}$ & $\begin{array}{c}\text { Air Mass } \\
\text { (3) }\end{array}$ & $\begin{array}{c}t_{\text {int }} \\
\text { (s) } \\
(4)\end{array}$ & $\begin{array}{c}\text { Calibrator Star } \\
\text { (5) }\end{array}$ & $\begin{array}{c}\text { Spectral Type } \\
\text { (6) }\end{array}$ \\
\hline 2MASS $0050-3322 \ldots \ldots \ldots . .$. & 2004 Sep 7 & 1.69 & 1440 & HD 225200 & $\mathrm{~A} 0 \mathrm{~V}$ \\
\hline 2MASS $0727+1710 \ldots \ldots \ldots \ldots$ & 2004 Mar 10 & 1.00 & 1280 & HD 56386 & A0 Vn \\
\hline 2MASS $0937+2931 \ldots \ldots \ldots \ldots$ & 2004 Mar 11 & 1.02 & 720 & HD 89239 & $\mathrm{~A} 0 \mathrm{~V}$ \\
\hline 2MASS 0939-2448 ........... & 2004 Mar 12 & 1.43 & 1080 & HD 81694 & $\mathrm{~A} 0 \mathrm{~V}$ \\
\hline SDSS $1110+0116 \ldots \ldots \ldots \ldots \ldots$ & 2004 Mar 11 & 1.14 & 1800 & HD 97585 & $\mathrm{~A} 0 \mathrm{~V}$ \\
\hline 2MASS $1114-2618 \ldots \ldots \ldots \ldots$ & 2004 Mar 12 & 1.75 & 1080 & HD 98949 & $\mathrm{~A} 0 \mathrm{~V}$ \\
\hline 2MASS $1217-0311 \ldots \ldots \ldots . .$. & 2004 Mar 11 & 1.13 & 720 & HD 109309 & $\mathrm{~A} 0 \mathrm{~V}$ \\
\hline SDSS $1346-0031 \ldots \ldots \ldots \ldots \ldots$ & 2004 Mar 12 & 1.09 & 720 & HD 116960 & $\mathrm{~A} 0 \mathrm{~V}$ \\
\hline SDSS $1624+0029 \ldots \ldots \ldots \ldots \ldots$ & 2004 Mar 12 & 1.06 & 720 & HD 136831 & $\mathrm{~A} 0 \mathrm{~V}$ \\
\hline 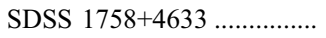 & 2004 Jul 23 & 1.22 & 720 & HD 158261 & $\mathrm{~A} 0 \mathrm{~V}$ \\
\hline
\end{tabular}

differences are seen in the relative brightness of the $K$-band flux peak and the shape of the $Y$-band peak. In particular, 2MASS $0937+2931$ exhibits weaker $K$-band emission and a broader $Y$-band flux peak compared to SDSS $1624+0029$, while 2 MASS $2228-4310$ has stronger $K$-band emission. Similar deviations are also seen among the three T7.5/T8 dwarfs-2MASS 0939-2448, 2MASS 1114-2618, and 2MASS 1217-0311—when compared to the similarly classified Gliese 570D.
What gives rise to these deviations? Shortward of the $Y$-band spectral peak, the dominant absorbers in T dwarf spectra are the pressure-broadened wings of the $\mathrm{K}_{\mathrm{I}}$ and $\mathrm{Na}$ I fundamental doublet lines centered at 0.77 and $0.59 \mu \mathrm{m}$, respectively ( Tsuji et al. 1999; Burrows et al. 2000; Allard et al. 2003; Burrows \& Volobuyev 2003). These features strengthen with later spectral type throughout the L and T dwarf sequences (Kirkpatrick et al. 1999; Reid et al. 2000; Burgasser et al. 2003a). The broad wings of the alkali
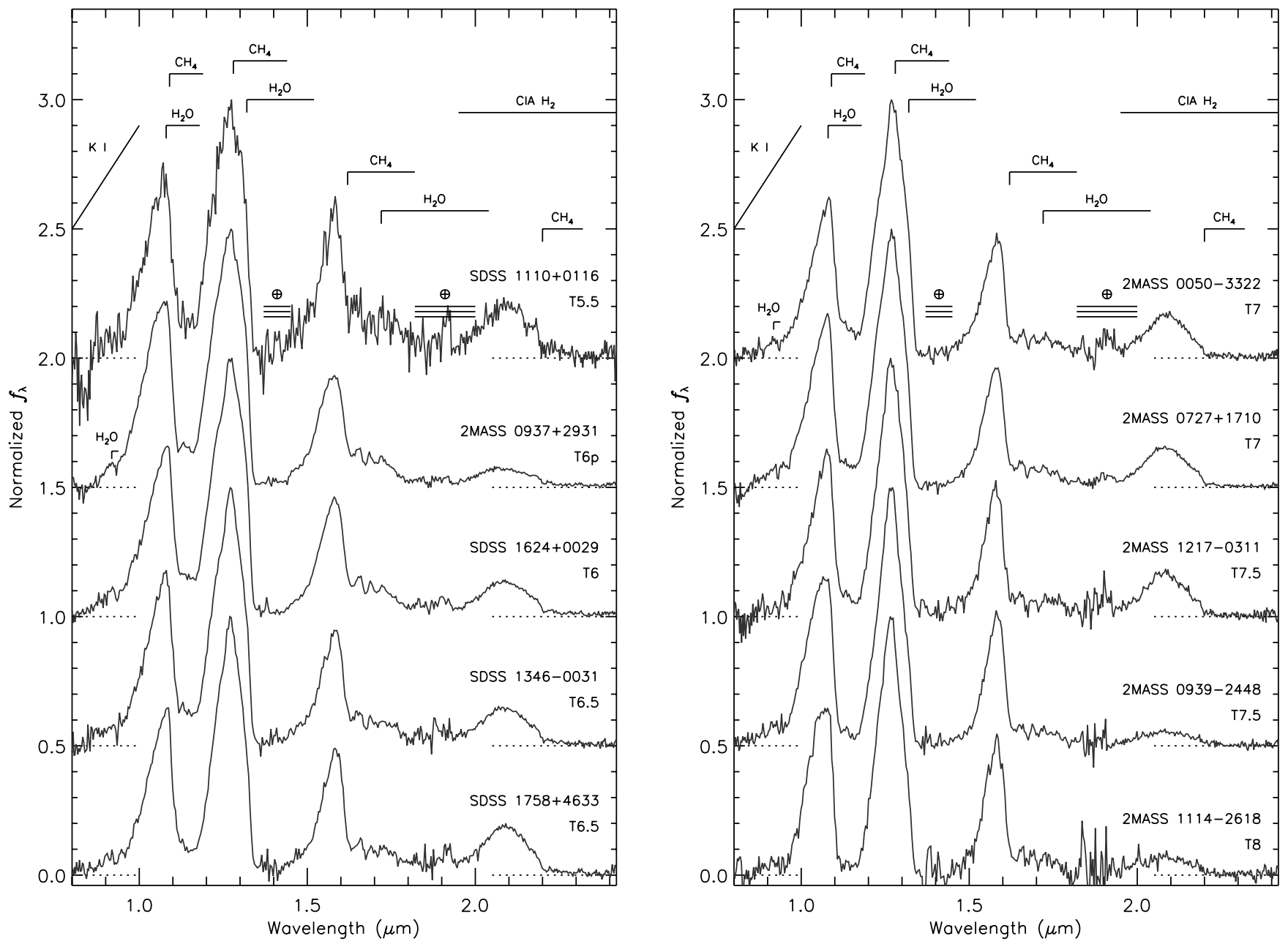

FIG. 1.-SpeX prism spectra for newly observed T dwarfs. All data are normalized at the $1.27 \mu \mathrm{m}$ flux peaks and offset by a constant (dotted lines). Major spectral features are labeled, and regions of strong telluric absorption are indicated by circled plus signs. 

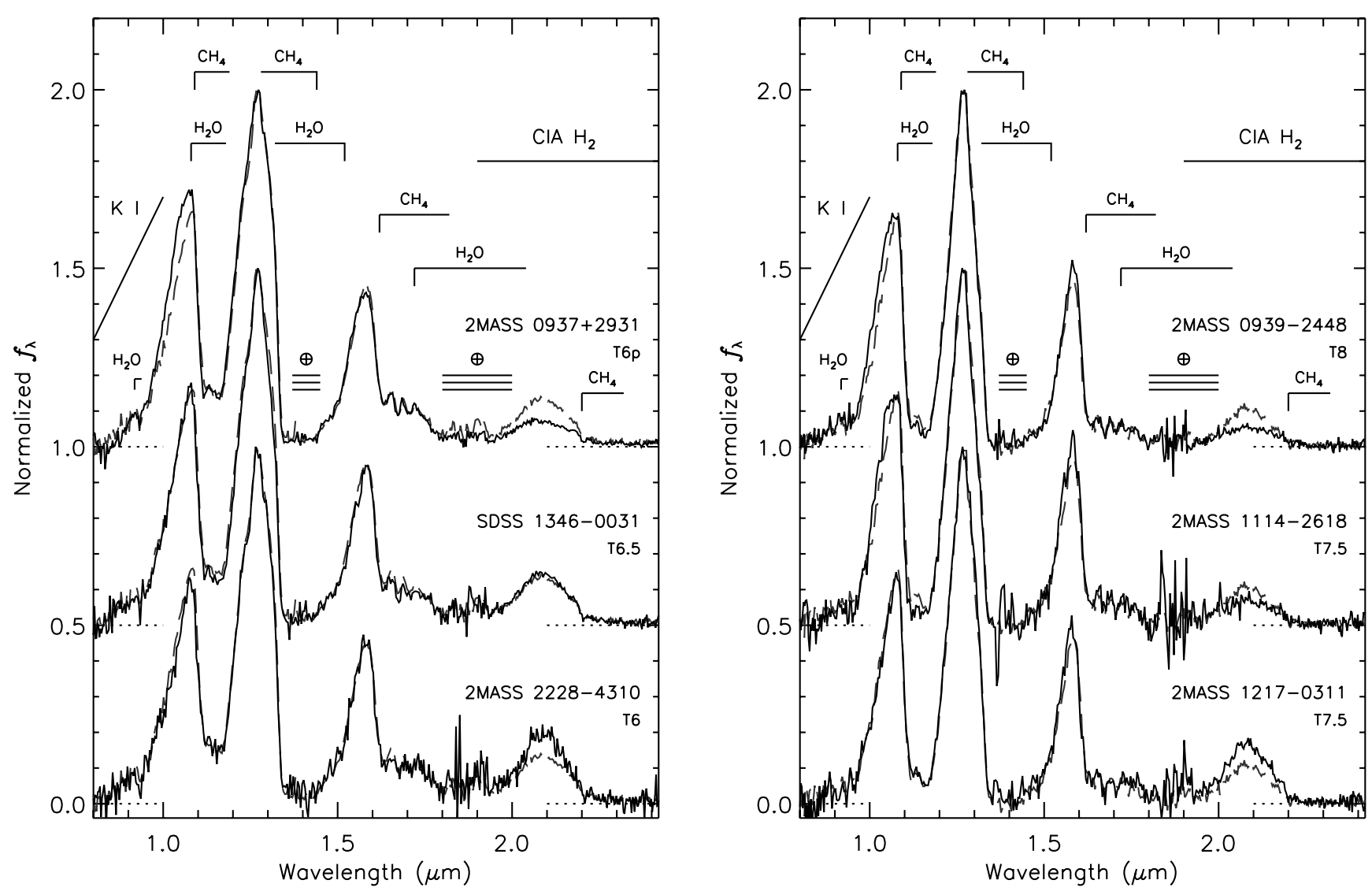

FIG. 2.-Surface gravity and metallicity features in T dwarf spectra. The left panel compares the normalized spectra of the T6-T6.5 dwarfs 2 MASS $0937+2931$, SDSS

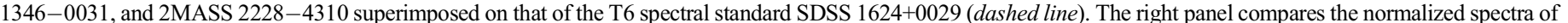

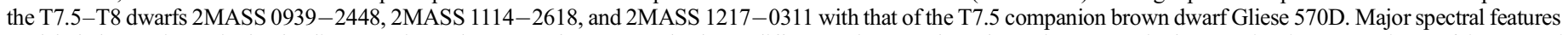

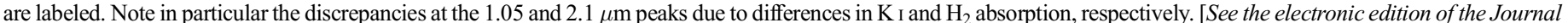
for a color version of this figure.]

lines, induced by kinematic perturbations by other chemical species (most importantly $\mathrm{H}_{2}$ and $\mathrm{He}$ ), are enhanced in higher pressure $(P)$ and higher density atmospheres. As atmospheric pressure scales as $d P / d \tau \sim P / \tau \propto g / \kappa_{\mathrm{R}}$ (where $\tau$ is the optical depth and $\kappa_{\mathrm{R}}$ is the Rosseland mean opacity), higher pressure photospheres $(\tau=2 / 3)$ are achieved in brown dwarfs with higher surface gravities and/or metal-deficient atmospheres (reduced $\kappa_{\mathrm{R}}$ ). For these sources, line-broadening theory (Allard et al. 2003; Burrows \& Volobuyev 2003) predicts the strongest absorption close to the line centers, resulting in steep $0.8-1.0 \mu \mathrm{m}$ spectral slopes due to the red wing of the $\mathrm{K}$ I doublet.

The $K$-band peak, while molded by $\mathrm{H}_{2} \mathrm{O}$ and $\mathrm{CH}_{4}$ bands at 1.8 and $2.2 \mu \mathrm{m}$, is dominated by another pressure-sensitive feature, collision-induced $\mathrm{H}_{2}$ absorption (Linsky 1969; Saumon et al. 1994; Borysow et al. 1997). The induced 1-0 quadrupolar moment of this molecule produces a broad, featureless absorption centered near $2.1 \mu \mathrm{m}$. Like the $\mathrm{K}$ I wings, $\mathrm{H}_{2}$ absorption arises from kinematic perturbations and is therefore enhanced in the higher pressure and higher density atmospheres present on high surface gravity and/or low-metallicity brown dwarfs.

While deviations in the strengths of the $\mathrm{K}_{\mathrm{I}}$ and $\mathrm{H}_{2}$ features have previously been linked to gravity and metallicity variations in T dwarfs (Burgasser et al. 2002b, 2003a; Burrows et al. 2002; Leggett et al. 2003; Knapp et al. 2004), Figure 2 demonstrates that these features are correlated. The steeper $\mathrm{K}$ I wings and enhanced $\mathrm{H}_{2}$ absorption exhibited in the spectra of 2MASS 0937+ 2931, 2MASS 0939-2448, and 2MASS 1114-2618 are both indicative of higher pressure photospheres, while the weaker $\mathrm{H}_{2}$ absorption in the spectra of 2MASS 2228-4310 and 2MASS 1217-0311 indicate low-pressure photospheres.

In contrast, the congruence of the $\mathrm{CH}_{4}$ and $\mathrm{H}_{2} \mathrm{O}$ bands for similarly classified $\mathrm{T}$ dwarfs suggests that gravity and metallicity effects for these features are minimal. The observed correlation between $T_{\text {eff }}$ and spectral type, the latter based on the strengths of the molecular bands, links $\mathrm{H}_{2} \mathrm{O}$ and $\mathrm{CH}_{4}$ to temperature. However, gas pressure does regulate the atmospheric abundance of $\mathrm{CH}_{4}$ and $\mathrm{H}_{2} \mathrm{O}$ in the principle reaction $\mathrm{CO}+3 \mathrm{H}_{2} \rightleftharpoons \mathrm{CH}_{4}+$ $\mathrm{H}_{2} \mathrm{O}$ (Fegley \& Lodders 1996; Burrows \& Sharp 1999), while metallicity modulates both $\mathrm{CH}_{4}$ and $\mathrm{H}_{2} \mathrm{O}$ abundances (Lodders $\&$ Fegley 2002). Hence, nearly all of the major absorption features in T dwarf spectra are affected in some manner by $T_{\text {eff }}, g$, and $[\mathrm{M} / \mathrm{H}]$.

\section{SPECTRAL MODELS}

To further investigate the physical origins of the spectral peculiarities described above, we have examined a new suite of brown dwarf spectral models that incorporate differences in $T_{\text {eff }}$, surface gravity, and metallicity. The models, developed by the Tucson group (Burrows et al. 2000, 2002, 2003, 2006), are self-consistent, nongray atmospheres incorporating up-to-date molecular opacities as described in Burrows et al. (2001). The atmospheres are assumed to be free of condensate dust species, consistent with prior modeling results (Tsuji et al. 1999; Allard et al. 2001), following the prescription of condensate rain-out as 


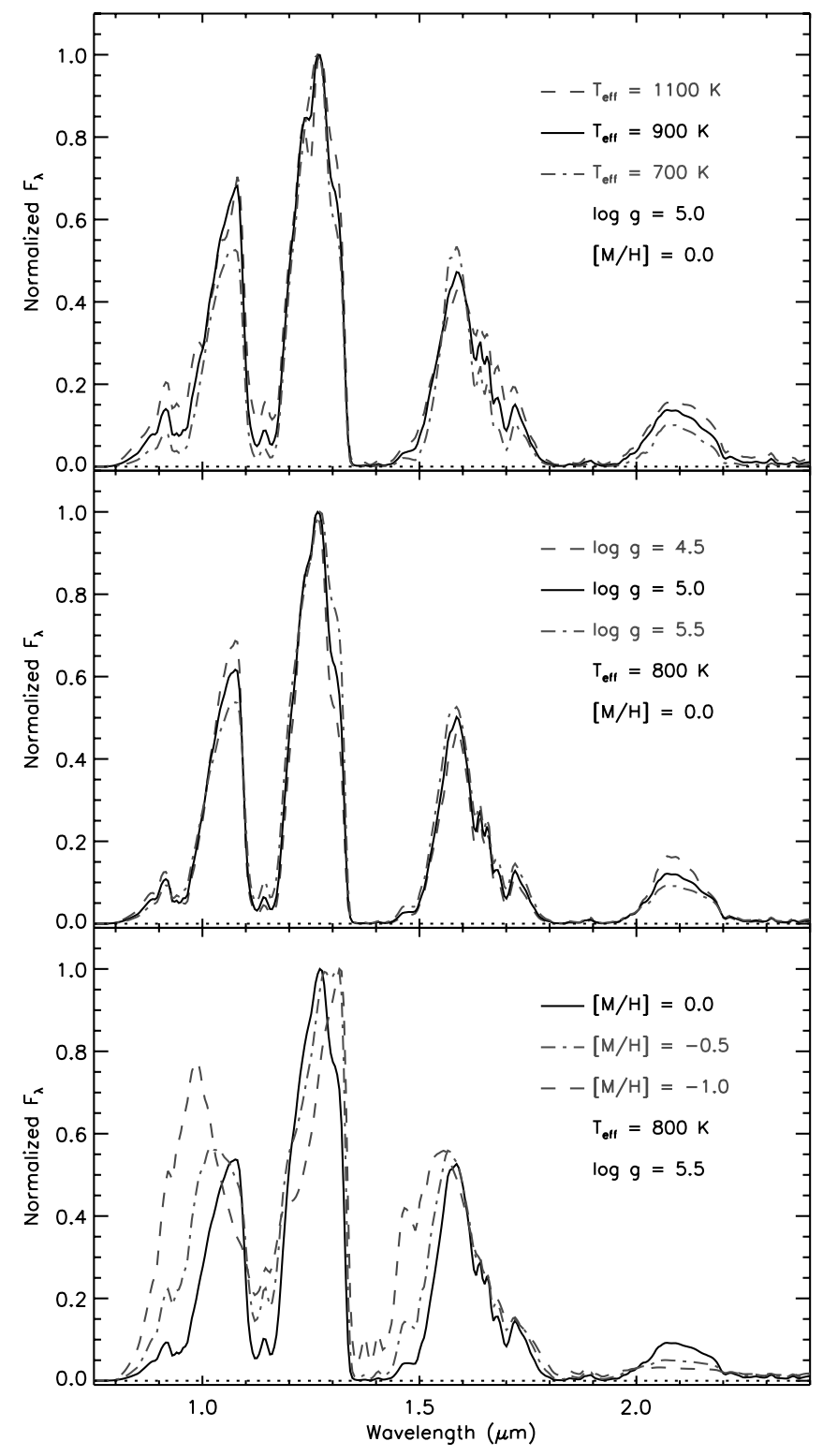

FIG. 3.-Comparison of temperature, surface gravity, and metallicity effects in $\mathrm{T}$ dwarf spectral models. The top panel displays three solar metallicity models with $\log g=5.0 \mathrm{~cm} \mathrm{~s}^{-2}$ and $T_{\mathrm{eff}}=1100$ (dashed line), 900 (solid line), and $700 \mathrm{~K}$ (dot-dashed line). The middle panel displays three solar metallicity models with $T_{\text {eff }}=800 \mathrm{~K}$ and $\log g=4.5$ (dashed line), 5.0 (solid line), and $5.5 \mathrm{~cm} \mathrm{~s}^{-2}$ (dotdashed line). The bottom panel displays three $T_{\text {eff }}=800 \mathrm{~K}, \log g=5.5 \mathrm{~cm} \mathrm{~s}^{-2}$ models with metallicities $[\mathrm{M} / \mathrm{H}]=0$ (solid line), -0.5 (dot-dashed line), and -1.0 (dashed line). Each spectral model has been deconvolved to the resolution of the SpeX data $(\lambda / \Delta \lambda \sim 150)$ and normalized at their $J$-band peak. [See the electronic edition of the Journal for a color version of this figure.]

described in Burrows \& Sharp (1999; see also Lodders 1999). Modified Lorentzian profiles with an ad hoc, smooth cutoff are used to model the line broadening of the $\mathrm{Na}$ I and $\mathrm{K}$ I doublets (Burrows et al. 2000). Nonequilibrium mixing effects (Saumon et al. 2003) are not considered. A full description of these models is given in Burrows et al. (2002).

Figure 3 compares three sequences of these spectral models, varying $T_{\text {eff }}, g$, and $[\mathrm{M} / \mathrm{H}]$, respectively. The spectral resolution of the models has been degraded using a Gaussian kernel to match the resolution of the SpeX prism data. There is general agreement in the overall spectral morphologies of the models and observed data; however, important discrepancies are pre- sent. Most prominent of these is the shape of the $1.6 \mu \mathrm{m} \mathrm{CH}_{4}$ band, reflecting continued deficiencies in the near-infrared opacities of this molecule for which only low-temperature $(300 \mathrm{~K})$ laboratory measurements have been obtained (Saumon et al. 2000; however, see Homeier et al. 2003). These opacities also detrimentally affect absorption features at 1.1 and $1.3 \mu \mathrm{m}$, although bands at 2.2 and $3.3 \mu \mathrm{m}$ have been found to be adequately reproduced (M. Cushing 2005, private communication). In addition, the line-broadening theory employed by these models predates more recent calculations by Burrows \& Volobuyev (2003), which predict a sharper cutoff for the red K I wing at $1.0 \mu \mathrm{m}$, in contrast to the modified Lorentzian profile used here, which is relatively flat over the $0.9-1.0 \mu \mathrm{m}$ waveband. As a result, alkali opacity at shorter wavelengths in the models is reduced (note the stronger $0.92 \mu \mathrm{m} \mathrm{H}_{2} \mathrm{O}$ as compared to the data),

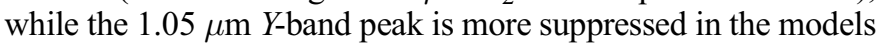
than observed.

The top panel of Figure 3 shows temperature variations for solar metallicity and $\log g=5.0 \mathrm{~cm} \mathrm{~s}^{-2}$ models. The trends in this sequence reflect those observed in $\mathrm{T}$ dwarf spectra as a function of spectral type, i.e., strengthening $\mathrm{H}_{2} \mathrm{O}$ and $\mathrm{CH}_{4}$ bands producing more acute triangular flux peaks, and stronger absorption shortward of $1 \mu \mathrm{m}$ and at $K$ band. The increasing depths of the molecular bands with cooler effective temperatures are largely a consequence of the increased column depth and therefore total opacity of the associated gas species.

The middle panel of Figure 3 compares surface gravity variations in the $T_{\text {eff }}=800 \mathrm{~K}$ solar metallicity models. Here, spectral variations are strongest at the $Y$ - and $K$-band flux peaks, although $\mathrm{H}_{2} \mathrm{O}$ and $\mathrm{CH}_{4}$ absorptions at 1.1 and $1.3 \mu \mathrm{m}$, and the $H$-band peak, are also affected. The $K$-band peak is suppressed in higher surface gravity models, consistent with the enhanced $\mathrm{H}_{2}$ absorption expected in higher pressure photospheres. On the other hand, surface gravity variations at the $Y$-band peak do not match the observed trends. The highest surface gravity model exhibits reduced $1.05 \mu \mathrm{m}$ flux relative to $1.27 \mu \mathrm{m}$, contrary to the brighter and broadened $Y$-band peaks seen in the empirical data. We attribute this discrepancy to the outdated alkali line-broadening profile employed in these models and leave analysis of this feature to a future study.

The similarity of surface gravity modulations of the $K$-band flux peak in the theoretical models to variations in the spectral data shown in Figure 2 is highlighted in Figure 4, which shows a similar sequence of T6/T6.5 and T7.5/T8 dwarfs but overlaid on the $T_{\text {eff }}=1000$ and $800 \mathrm{~K}$ solar metallicity models, respectively. Inequities in the $1.6 \mu \mathrm{m} \mathrm{CH}_{4}$ band notwithstanding, the spectral models reproduce reasonably well the relative variations observed in the $K$-band flux peaks, although both 2MASS $0937+2931$ and 2MASS 0939-2448 exhibit stronger $K$-band suppression than the highest surface gravities permitted by the models.

The bottom panel of Figure 3 compares metallicity effects at fixed surface gravity $(\log g=5.5)$ and temperature $\left(T_{\text {eff }}=\right.$ $800 \mathrm{~K}$ ) for $[\mathrm{M} / \mathrm{H}]=0,-0.5$, and -1 . The higher surface gravity examined here is appropriate for old brown dwarf members of the metal-poor Galactic thick disk and halo populations. Spectral variations are far more extreme in this case. The $K$-band peak is suppressed at lower metallicities, as expected for enhanced $\mathrm{H}_{2}$ absorption. At the same time, emergent flux appears to be relatively enhanced shortward of $1 \mu \mathrm{m}$, due to reduced $\mathrm{Na}$ and $\mathrm{K}$ abundances and their corresponding opacities. The specific shape of the spectrum at these wavelengths should be treated with caution, however, given the older line-broadening theory used in the models. Shifts in the $J$ - and $H$-band peak wavelengths 

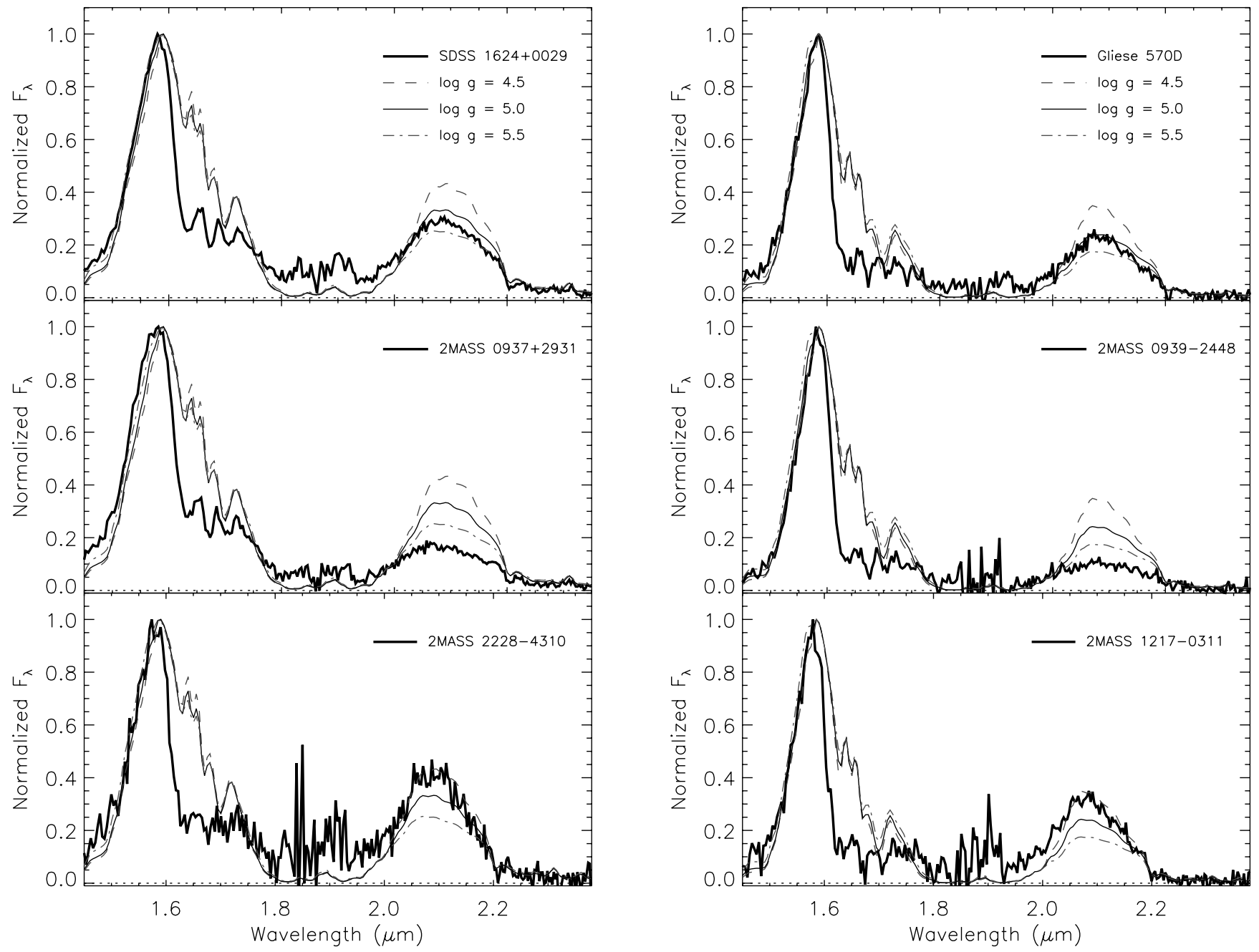

Fig. 4. - Comparison of models to spectra in the 1.5-2.4 $\mu \mathrm{m}$ spectral region. Shown are data (solid lines) for the T6-T6.5 dwarfs SDSS 1624+0029, 2MASS 0937+2931, and 2MASS 2228-4310 (left panels) and the T7.5-T8 dwarfs Gliese 570D, 2MASS 0939-2448, and 2MASS $1217-0311$ (right panels). Resampled solar metallicity spectral models for $\log g=4.5$ (dashed line), 5.0 (solid line), and $5.5 \mathrm{~cm} \mathrm{~s}^{-2}$ (dot-dashed line); $T_{\text {eff }}=800$ (left panels) and $1000 \mathrm{~K}$ (right panels) are overplotted. Both data and models are normalized at the $H$-band peak. [See the electronic edition of the Journal for a color version of this figure.]

are due to reduced $\mathrm{H}_{2} \mathrm{O}$ abundances and increased collisioninduced $\mathrm{H}_{2}$ absorption extending into the $H$ band. Overall, these spectral variations are more substantial than those seen in the empirical data, a sign that significant subsolar metallicities $([\mathrm{M} / \mathrm{H}] \lesssim$ -0.5 ) are not present among the T dwarfs examined here. However, the broadened $Y$-band peaks and strong $K$-band suppression in the spectra of 2MASS 0937+2931, 2MASS 0939-2448, and 2MASS 1114-2618 do hint at slightly subsolar metallicities for these sources.

\section{MEASURING PHYSICAL PROPERTIES FROM T DWARF SPECTRA}

\subsection{The Method}

The spectral models confirm that the pressure-sensitive $\mathrm{H}_{2}$ and $\mathrm{K}$ I absorptions are more strongly influenced by surface gravity effects than the absorption bands of $\mathrm{H}_{2} \mathrm{O}$ and $\mathrm{CH}_{4}$, while the latter vary more strongly with $T_{\text {eff }}$, at least for the temperature regime considered here. By contrasting the strengths of these features, it should be possible in principle to extract the effective temperatures and surface gravities of these objects. In practice, this pursuit has proven problematic due to persistent inadequacies in molecular opacities and corresponding systematic errors in the models (e.g., Burgasser et al. 2004a). What is required is a means of calibrating the spectral models using one or more empirical fiducials.

Fortunately, such a fiducial exists in the T dwarf Gliese 570D (Burgasser et al. 2000). This widely separated $\left(\rho=258^{\prime \prime}=\right.$ $1530 \mathrm{AU})$, common proper motion brown dwarf companion to the nearby $(5.91 \pm 0.06 \mathrm{pc}$; ESA 1997) Gliese $570 \mathrm{ABC}$ system has both distance and luminosity measurements that are empirically well constrained. The age of this system is estimated to be 2-5 Gyr based on a comparison of age, activity, and kinematic relations for the $\mathrm{K}$ and $\mathrm{M}$ stellar components (Burgasser et al. 2000; Geballe et al. 2001). The K4 V primary has a near-solar metallicity $([\mathrm{Fe} / \mathrm{H}]=0.00-0.16$; Feltzing \& Gustafsson 1998; Thoren \& Feltzing 2000; Allende Prieto \& Lambert 2000). Assuming coevality, Gliese $570 \mathrm{D}$ is one of the few T dwarfs with both age and metallicity constraints. Geballe et al. (2001) derived fairly precise temperature $\left(T_{\text {eff }}=784-824 \mathrm{~K}\right)$ and surface gravity $\left[g=(1-2) \times 10^{5} \mathrm{~cm} \mathrm{~s}^{-2}\right]$ estimates, the former based on the measured luminosity and a model-dependent radius, the latter based on brown dwarf evolutionary models (Burrows et al. 1997).

Using Gliese 570D as our empirical fiducial, our procedure was then as follows. The strengths of the major $\mathrm{H}_{2} \mathrm{O}$ bands and relative fluxes of the spectral peaks were measured for both the 
TABLE 3

Spectral Ratios

\begin{tabular}{|c|c|c|c|c|c|c|}
\hline $\begin{array}{l}\text { Source } \\
\text { (1) }\end{array}$ & $\begin{array}{c}\text { Spectral Type } \\
\text { (2) }\end{array}$ & $\begin{array}{l}\mathrm{H}_{2} \mathrm{O}-J \\
\text { (3) }\end{array}$ & $\begin{array}{l}\mathrm{H}_{2} \mathrm{O}-H \\
\quad \text { (4) }\end{array}$ & $\begin{array}{l}K / J \\
(5)\end{array}$ & $\begin{array}{c}K / H \\
(6)\end{array}$ & $\begin{array}{l}Y / J \\
(7)\end{array}$ \\
\hline 2MASS $0034+0523 \ldots \ldots \ldots$ & T6.5 & 0.103 & 0.229 & 0.100 & 0.218 & 0.456 \\
\hline 2MASS $0050-3322 \ldots \ldots .$. & $\mathrm{T} 7$ & 0.104 & 0.266 & 0.180 & 0.388 & 0.363 \\
\hline 2MASS $0243-2453 \ldots \ldots .$. & T6 & 0.145 & 0.297 & 0.197 & 0.406 & 0.444 \\
\hline 2MASS $0415-0935 \ldots \ldots .$. & $\mathrm{T} 8$ & 0.041 & 0.183 & 0.131 & 0.255 & 0.382 \\
\hline 2MASS $0727+1710 \ldots \ldots \ldots$ & $\mathrm{T} 7$ & 0.085 & 0.224 & 0.164 & 0.351 & 0.427 \\
\hline 2MASS $0937+2931 \ldots \ldots \ldots$. & T6p & 0.151 & 0.316 & 0.076 & 0.174 & 0.539 \\
\hline 2MASS 0939-2448 ......... & T8 & 0.038 & 0.149 & 0.059 & 0.117 & 0.493 \\
\hline SDSS $1110+0116 \ldots \ldots \ldots \ldots$ & T5.5 & 0.152 & 0.303 & 0.217 & 0.379 & 0.497 \\
\hline 2MASS $1114-2618 \ldots \ldots \ldots$ & T7.5 & 0.039 & 0.177 & 0.076 & 0.150 & 0.482 \\
\hline 2MASS $1217-0311 \ldots \ldots .$. & T7.5 & 0.066 & 0.207 & 0.179 & 0.366 & 0.374 \\
\hline 2 MASS $1231+0847 \ldots \ldots \ldots$ & T5.5 & 0.181 & 0.271 & 0.157 & 0.328 & 0.451 \\
\hline SDSS $1346-0031 \ldots \ldots \ldots \ldots$ & T6.5 & 0.131 & 0.278 & 0.156 & 0.351 & 0.430 \\
\hline Gliese 570D ....................... & $\mathrm{T} 7.5$ & 0.063 & 0.198 & 0.116 & 0.253 & 0.397 \\
\hline SDSS $1624+0029 \ldots \ldots \ldots . .$. & $\mathrm{T} 6$ & 0.154 & 0.280 & 0.142 & 0.311 & 0.422 \\
\hline SDSS $1758+4633 \ldots \ldots \ldots \ldots$ & T6.5 & 0.101 & 0.247 & 0.200 & 0.411 & 0.400 \\
\hline 2MASS $2228-4310 \ldots \ldots .$. & $\mathrm{T} 6$ & 0.157 & 0.293 & 0.204 & 0.440 & 0.383 \\
\hline 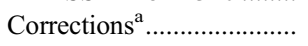 & $\ldots$ & 1.173 & 1.567 & 0.952 & 1.064 & 0.883 \\
\hline
\end{tabular}

${ }^{\text {a }}$ Scale factors applied to model ratios to bring them into agreement with measurements for Gliese 570D, assuming $T_{\text {eff }}=800 \mathrm{~K}$ and $\log g=5.1$; see $\S 4.1$.

empirical data sample and theoretical models using the following ratios:

$$
\begin{gathered}
\mathrm{H}_{2} \mathrm{O}-J=\frac{\int F_{1.14-1.165}}{\int F_{1.26-1.285}} \\
\mathrm{H}_{2} \mathrm{O}-H=\frac{\int F_{1.48-1.52}}{\int F_{1.56-1.60}} \\
Y / J=\frac{\int F_{1.005-1.045}}{\int F_{1.25-1.29}} \\
K / J=\frac{\int F_{2.06-2.10}}{\int F_{1.25-1.29}} \\
K / H=\frac{\int F_{2.06-2.10}}{\int F_{1.56-1.60}}
\end{gathered}
$$

where $\int F_{\lambda_{1}-\lambda_{2}}$ denotes the integrated flux between wavelengths $\lambda_{1}$ and $\lambda_{2}$ (in microns). The first two ratios are identical to those defined for the near-infrared classification of $\mathrm{T}$ dwarfs; the spectral region sampled by these is shown in Figure 5 of Burgasser et al. (2006). Note that ratios sampling the poorly modeled $\mathrm{CH}_{4}$ bands are not considered here. The $K / J$ ratio has also been used previously to examine variations in $\mathrm{H}_{2}$ absorption (Burgasser et al. 2004b, 2006). The $Y / J$ and $K / H$ color ratios are defined here for the first time. Measurements of these ratios on the empirical data are given in Table 3 .

A series of $\mathrm{H}_{2} \mathrm{O}-J$ and $K / H$ ratios for the solar metallicity models are shown in Figure 5. Both ratios vary according to differences in $T_{\text {eff }}$ and $\log g$, although gravity variations are stronger in the $K / H$ color ratio. Gravity variations also affect the two ratios in opposite ways: higher gravity models yield large $\mathrm{H}_{2} \mathrm{O}-J$ values (implying weaker absorption) and smaller $K / H$ values (implying weaker $K$-band emission). Similar trends are seen with the $\mathrm{H}_{2} \mathrm{O}-H$ and $K / J$ ratios, respectively. All of these trends are consistent with the qualitative properties of the model spectra shown in Figure 3.

Calibration of the model ratios was achieved by correcting these values to those measured from the SpeX spectrum of
Gliese 570D. Adopting $T_{\text {eff }}=800 \mathrm{~K}$ and $\log g=5.1$ for this source, we computed the corresponding model ratios by linear interpolation. Correction factors, listed at the bottom of Table 3, were defined as the ratio of the spectral data measurement to the model value. For four of the ratios, model values differ by less than $20 \%$; the $\mathrm{H}_{2} \mathrm{O}-\mathrm{H}$ index, on the other hand, requires a $60 \%$ correction. The correction factors were applied to all of the solar metallicity model ratios, regardless of $T_{\text {eff }}$ or gravity, and therefore represents a first-order calibration of the models.

In principle, any of the ratios defined in equations (1)-(5) could be used for comparison to the spectral data. We restrict our primary analysis to the $\mathrm{H}_{2} \mathrm{O}-J$ index, which requires a correction factor smaller than $\mathrm{H}_{2} \mathrm{O}-H$, and the $K / H$ index, which gives a quantitative measure of the behavior demonstrated in Figure 4. The corrected model ratios were resampled in steps of $20 \mathrm{~K}$ in $T_{\text {eff }}$ and $0.1 \mathrm{dex}$ in $\log g$ by linear interpolation. Then, for each spectrum, we identified the region in $T_{\text {eff }}$ and $\log g$ phase space for which the corrected model ratios agreed with the empirical ratios, assuming a $10 \%$ uncertainty (see $\S 4.3$ ).

Figures 6 and 7 illustrate these matches. The $\mathrm{H}_{2} \mathrm{O}-J$ and $K / H$ ratios each constrain a set of $T_{\text {eff }}$ and $\log g$ values that span the model parameter space diagonally; e.g., agreement in the $\mathrm{H}_{2} \mathrm{O}-J$ ratios span low temperatures and high surface gravities to high temperatures and low surface gravities. The $K / H$ ratios match an orthogonal phase space. The intersection of these phase spaces provides an unambiguous constraint on both $T_{\text {eff }}$ and $\log g$.

\subsection{Results \\ 4.2.1. $T_{\mathrm{eff}}$ and $g$ Estimates}

Table 4 lists the ranges of $T_{\text {eff }}$ and $\log g$ constrained by the two ratios for each source in our sample. For 13 of the 16 sources (including Gliese 570D), these values are well defined, with typical uncertainties of $40-60 \mathrm{~K}$ in $T_{\text {eff }}$ and $0.1-0.3 \mathrm{dex}$ in $\log g$. For three of the T dwarfs-2MASS 0937+2931, 2MASS 0939-2448, and 2MASS 1114-2618 - no phase space intersection was found. The case of 2MASS $0937+2931$ is discussed in further detail below. For 2MASS 0939-2448 and 2MASS 1114-2618, close examination of Figures 6 and 7 suggests that phase-space intersections are possible at lower $T_{\text {eff }}$ values than those spanned by our model 

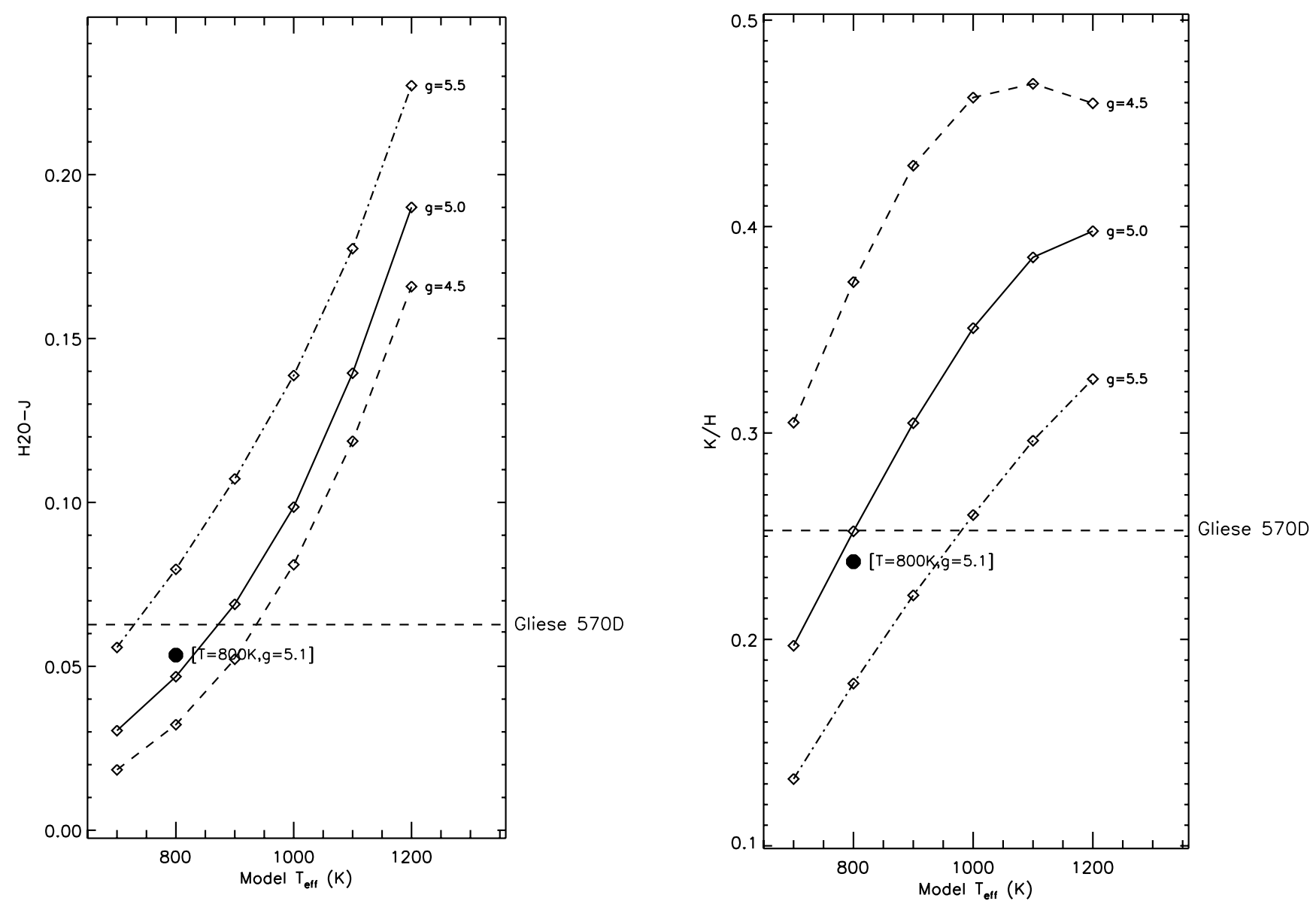

FIG. 5.-Values for the spectral ratios $\mathrm{H}_{2} \mathrm{O}-J$ and $K / H$, as measured on solar metallicity models (points with dot-dashed lines connecting constant surface gravity models). Values measured on the spectrum of Gliese 570D are indicated by the dashed lines. The solid circle indicates the interpolated model index value for the adopted physical parameters of Gliese $570 \mathrm{D}, T_{\text {eff }}=800 \mathrm{~K}, \log g=5.1 \mathrm{~cm} \mathrm{~s}^{-2}$, and $[\mathrm{M} / \mathrm{H}]=0$.

set; i.e., for $T_{\text {eff }} \lesssim 700 \mathrm{~K}$. This is intriguing, since we derive $T_{\text {eff }}=740-760 \mathrm{~K}$ for the T8 2MASS 0415-0935, currently the coldest and lowest luminosity brown dwarf known (Burgasser et al. 2002b; Golimowski et al. 2004; Vrba et al. 2004). Parallax measurements can determine whether 2MASS 0939-2448 and 2MASS 1114-2618 are in fact colder and fainter brown dwarfs.

Are these temperatures and surface gravities reasonable? Eight of the T dwarfs in our sample have prior $T_{\text {eff }}$ determinations from Golimowski et al. (2004) and Vrba et al. (2004), based on parallax and bolometric luminosity $(L)$ measurements; these values are listed in Table 4 . In all eight cases, our derived $T_{\text {eff }}$ values are consistent. This agreement may have much to do with the large $T_{\text {eff }}$ estimate ranges from Golimowski et al. (2004) and Vrba et al. (2004), as high as $300 \mathrm{~K}$, due to uncertainties in the radii adopted to compute $T_{\text {eff }}$ from $L$. Our $T_{\text {eff }}$ estimates are typically in the middle or high end of the ranges from these studies. The only surface gravity estimates for field $\mathrm{T}$ dwarfs reported to date are those of Knapp et al. (2004), based on a comparison of near-infrared colors to atmosphere models by Marley et al. (2002). In this case, we find that our estimates are systematically $0.3-0.5$ dex higher than the Knapp et al. values. As the latter are stated without uncertainties, we simply point out this discrepancy for further study.

\subsubsection{A Subsolar Metallicity for 2MASS 0937+2931}

The parameter spaces for 2MASS $0937+2931$ do not intersect in Figure 6, but it appears that they would if higher surface gravities were modeled. However, surface gravities larger than $\log g=5.5$ are restricted by the interior physics (Burrows et al. 1997). An alternate hypothesis is that the spectrum is influenced by a third parameter, namely, metallicity.

We can quantitatively test this case by introducing metallicity variations into the model set. Applying the same corrections to the model ratios as above (i.e., assuming Gliese 570D has $[\mathrm{M} / \mathrm{H}]=0$ ), linearly interpolating between the $[\mathrm{M} / \mathrm{H}]=0$ and -0.5 models in 0.1 dex steps for $700 \leq T_{\text {eff }} \leq 1200 \mathrm{~K}$ and $5.0 \leq$ $\log g \leq 5.5$, and performing the same comparative analysis, we derive the series of parameter phase spaces (effectively, slices of a three-dimensional parameter phase volume) shown in Figure 8. We find that, for the case of 2 MASS $0937+2931$, the phase spaces intersect for metallicities $-0.1 \leq[\mathrm{M} / \mathrm{H}] \leq-0.4,780 \leq T_{\text {eff }} \leq$ 860 , and $5.0 \leq \log g \leq 5.5$. Lower metallicities may also be feasible at lower surface gravities. One other source in our sample, 2MASS 0034+0523, also exhibits intersecting parameter spaces for slightly subsolar metallicities, $-0.2 \leq[\mathrm{M} / \mathrm{H}] \leq 0$. This object has also been noted for its strong $K$-band suppression (Burgasser et al. 2004b).

Our analysis represents the first quantitative constraints of metallicity for a brown dwarf and are consistent with prior qualitative conclusions. However, the derived values should be treated with caution for reasons other than the fidelity of the theoretical models. Constraining the three parameters $T_{\text {eff }}, g$, and $[\mathrm{M} / \mathrm{H}]$ cannot be done unambiguously using only two spectral ratios; at least one additional constraint is required. A promising candidate is the $Y / J$ ratio, as the metal-poor models shown in Figure 5 exhibit 

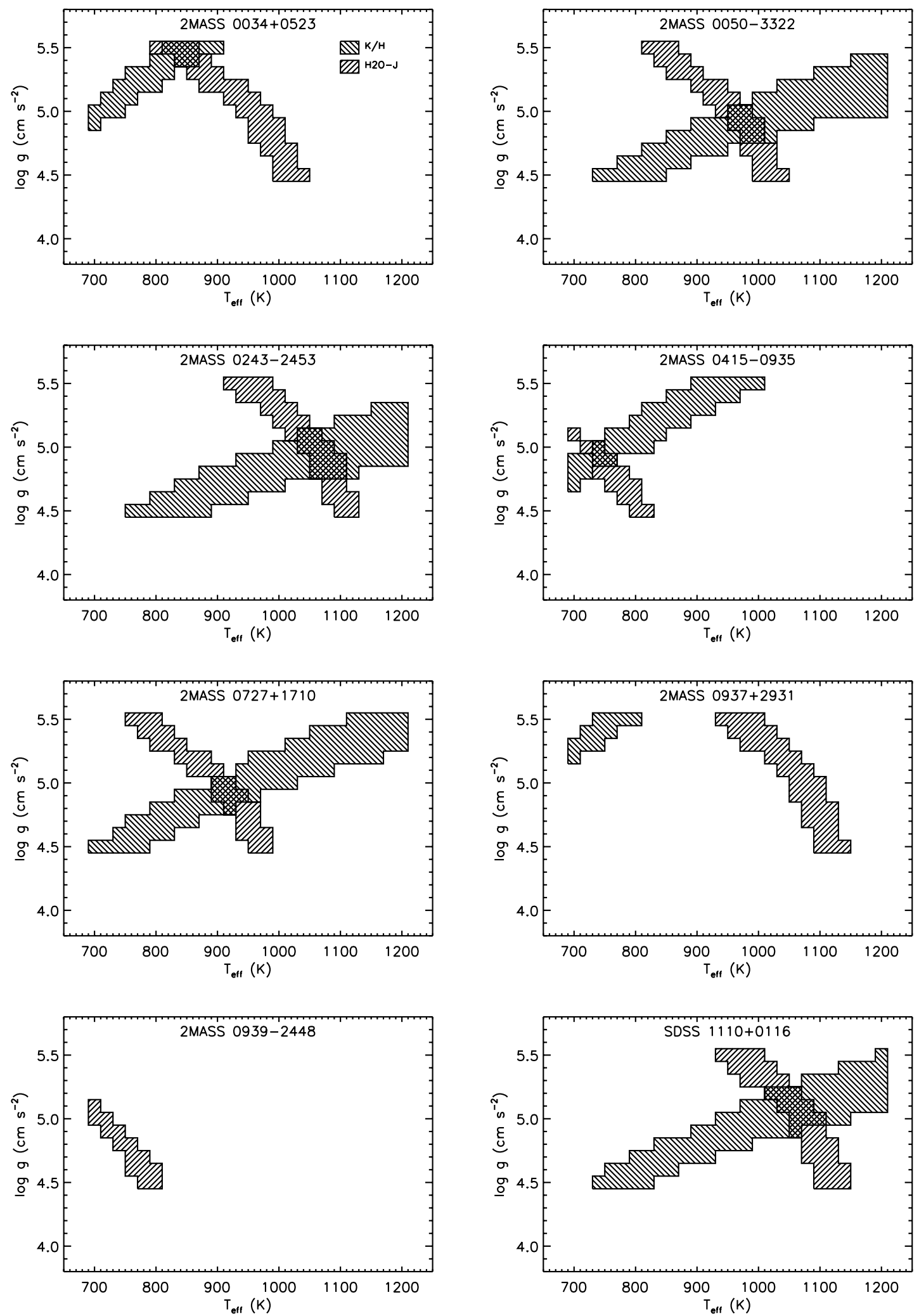

Fig. 6.-Derived physical parameter phase spaces for the T dwarfs 2MASS 0034+0523, 2MASS 0050-3322, 2MASS 0243-2453, 2MASS 0415-0935, 2MASS $0727+1710$, 2MASS $0937+2931$, 2MASS 0939-2448, and SDSS $1110+0116 . T_{\text {eff }}$ and $\log g$ values for which measurements of the spectral ratios $\mathrm{H}_{2} \mathrm{O}-J$ and $K / H$ match scaled values for the models (assuming an uncertainty of $10 \%$ ) are indicated by hatched regions. The overlap cross-hatched regions represent our best match "fits" for these parameters. 

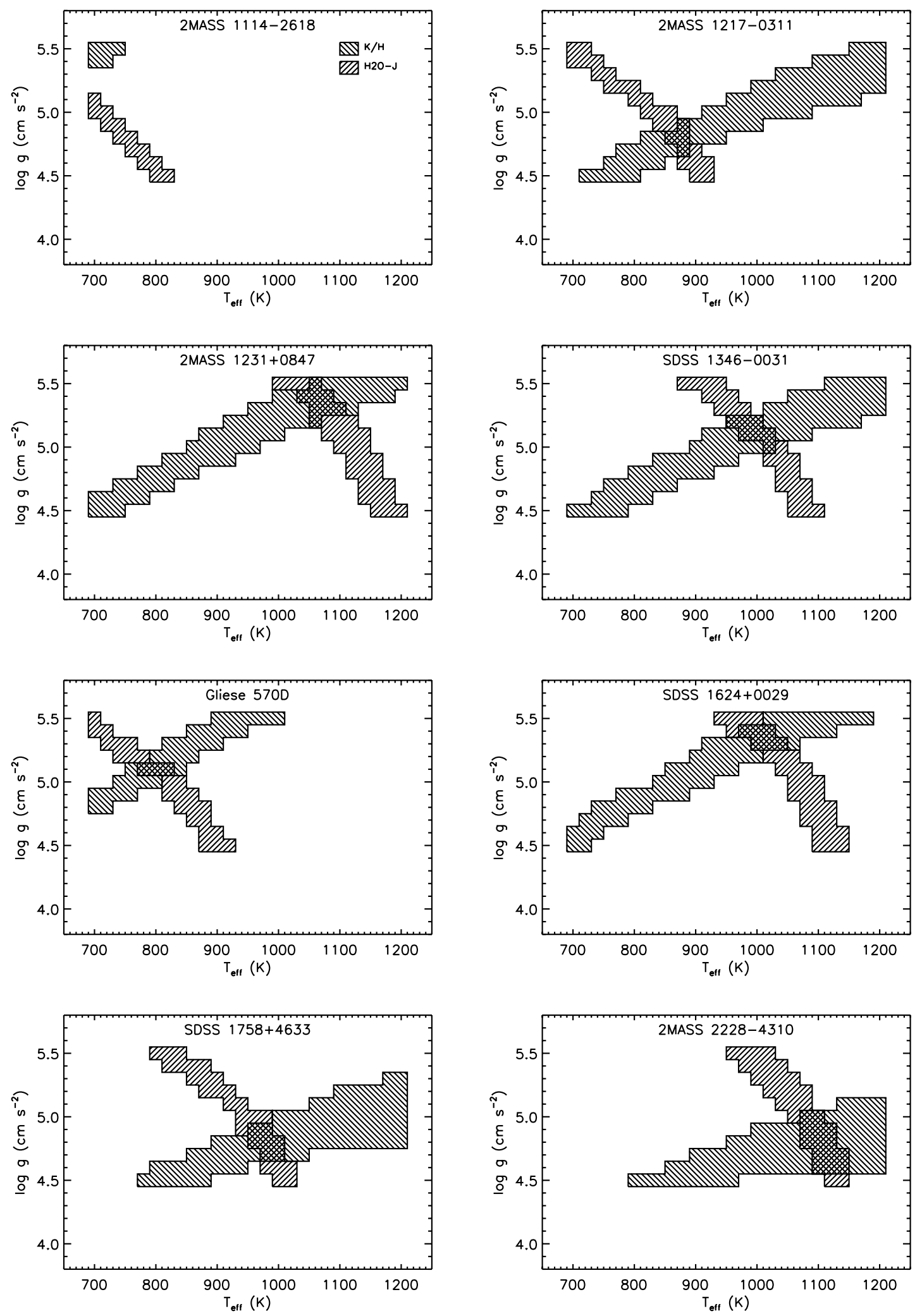

FIG. 7.-Same as Fig. 6, but for the T dwarfs 2MASS 1114-2618, 2MASS 1217-0311, 2MASS 1231+0847, SDSS 1346-0031, Gliese 570D, SDSS 1624+0029, SDSS $1758+4633$, and 2MASS $2228-4310$. 
TABLE 4

Derived Physical Parameters for T Dwarfs

\begin{tabular}{|c|c|c|c|c|c|c|c|}
\hline \multirow[b]{2}{*}{$\begin{array}{l}\text { SourCE } \\
\text { (1) }\end{array}$} & \multirow[b]{2}{*}{$\begin{array}{c}\text { Spectral TyPE } \\
\text { (2) }\end{array}$} & \multirow[b]{2}{*}{$\begin{array}{c}T_{\text {eff }} \\
(\mathrm{K}) \\
(3)\end{array}$} & \multirow[b]{2}{*}{$\begin{array}{c}\log g \\
\left(\mathrm{~cm} \mathrm{~s}^{-2}\right) \\
(4)\end{array}$} & \multirow[b]{2}{*}{$\begin{array}{c}{[\mathrm{M} / \mathrm{H}]} \\
(5)\end{array}$} & \multicolumn{3}{|c|}{ Published Values } \\
\hline & & & & & $\begin{array}{c}T_{\text {eff }}^{\mathrm{a}} \\
(\mathrm{K}) \\
(6)\end{array}$ & $\begin{array}{l}T_{\text {eff }}^{\mathrm{b}} \\
(\mathrm{K}) \\
(7)\end{array}$ & $\begin{array}{c}\log g^{\mathrm{c}} \\
\left(\mathrm{cm} \mathrm{s}^{-2}\right) \\
(8)\end{array}$ \\
\hline 2MASS $0034+0523 \ldots \ldots \ldots \ldots$ & T6.5 & $820-860$ & $5.4-5.5$ & $-0.2 \sim 0$ & $\ldots$ & $\ldots$ & $\ldots$ \\
\hline 2MASS $0050-3322 \ldots \ldots \ldots . .$. & $\mathrm{T} 7$ & $960-1000$ & $4.8-5.0$ & 0 & $\ldots$ & $\ldots$ & $\ldots$ \\
\hline 2MASS $0243-2453 \ldots \ldots \ldots .$. & T6 & $1040-1100$ & $4.8-5.1$ & 0 & $825-1150$ & $950-1170$ & 4.5 \\
\hline 2MASS $0415-0935 \ldots \ldots \ldots . .$. & $\mathrm{T} 8$ & $740-760$ & $4.9-5.0$ & 0 & $600-750$ & $690-850$ & 5.0 \\
\hline 2MASS $0727+1710 \ldots \ldots \ldots \ldots$ & $\mathrm{T} 7$ & $900-940$ & $4.8-5.0$ & 0 & $725-950$ & $830-1020$ & 4.5 \\
\hline 2MASS $0937+2931 \ldots \ldots \ldots \ldots$ & T6p & $780-840^{\mathrm{d}}$ & $5.3-5.5^{\mathrm{d}}$ & $-0.4 \sim-0.1$ & $725-1000$ & $700-850$ & 5.5 \\
\hline 2MASS 0939-2448 .......... & $\mathrm{T} 8$ & $\lesssim 700$ & $\ldots$ & $\ldots$ & $\ldots$ & $\ldots$ & $\ldots$ \\
\hline SDSS $1110+0116 \ldots \ldots \ldots \ldots \ldots$ & T5.5 & $1020-1100$ & $4.9-5.2$ & 0 & $\ldots$ & $\ldots$ & 4.5 \\
\hline 2MASS $1114-2618 \ldots \ldots \ldots . .$. & $\mathrm{T} 7.5$ & $\lesssim 700$ & $\ldots$ & $\ldots$ & $\ldots$ & $\ldots$ & $\ldots$ \\
\hline 2MASS $1217-0311 \ldots \ldots \ldots . .$. & $\mathrm{T} 7.5$ & $860-880$ & $4.7-4.9$ & 0 & $725-925$ & $820-1000$ & 4.5 \\
\hline 2MASS $1231+0847 \ldots \ldots \ldots \ldots$ & T5.5 & $1040-1100$ & $5.2-5.5$ & 0 & $\ldots$ & $\ldots$ & 5.0 \\
\hline SDSS $1346-0031 \ldots \ldots \ldots \ldots . . . . . .$. & T6.5 & $960-1020$ & $5.0-5.2$ & 0 & $875-1200$ & $950-1180$ & 4.5 \\
\hline Gliese 570D .......................... & $\mathrm{T} 7.5$ & $780-820$ & 5.1 & 0 & $784-824^{\mathrm{e}}$ & $\ldots$ & $5.0-5.3^{\mathrm{e}}$ \\
\hline SDSS $1624+0029 \ldots \ldots \ldots \ldots . . .$. & T6 & $980-1040$ & $5.3-5.4$ & 0 & $800-1100$ & $920-1100$ & 5.0 \\
\hline SDSS $1758+4633 \ldots \ldots \ldots \ldots . . . .$. & T6.5 & $960-1000$ & $4.7-4.9$ & 0 & $\ldots$ & $\ldots$ & 4.5 \\
\hline 2MASS $2228-4310 \ldots \ldots \ldots . .$. & T6 & $1080-1140$ & $4.6-5.0$ & 0 & $\ldots$ & $\ldots$ & $\ldots$ \\
\hline
\end{tabular}

$\begin{array}{ll}\text { a } & T_{\text {eff }} \text { from Golimowski et al. (2004). } \\ \text { b } & T_{\text {eff }} \text { from Vrba et al. (2004). } \\ \text { c } & \log g \text { from Knapp et al. (2004). } \\ \text { d } & T_{\text {eff }} \text { and } \log g \text { for }[\mathrm{M} / \mathrm{H}]=-0.2 \text {. See } \S 4.2 .2 \text {. } \\ \text { e } & T_{\text {eff }} \text { and } \log g \text { from Geballe et al. }(2001) .\end{array}$

significant variations at these short wavelengths. We defer more thorough examination of this third index until such time as the current generation of spectral models incorporate a more rigorous line-broadening theory.

\subsection{Assessing Systematic Effects}

While the $T_{\text {eff }}$ and $\log g$ estimates made here are reasonable and generally consistent with prior work, it is important to identify and characterize any source of systematic error that may skew the results. Such systematic effects can arise from the calibration or quality of the spectral data, the choice and calibration of the spectral ratios used, and limitations of the spectral models themselves. We examine these effects here in detail.

\subsubsection{Flux Calibration of the Spectral Data}

Accurate measurement of color ratios such as $K / H$ assumes that the spectral data portray the true color of the source, which brings into question possible reddening of the observed spectrum and the accuracy of the overall flux calibration. Interstellar reddening can generally be ruled out for our sample, as all of the sources lie at distances of $\sim 20$ pc or less. A more local source of reddening, differential color refraction through our atmosphere, has been mitigated by observing the sources with the slit aligned at the parallactic angle. We therefore assume that both of these effects are minimal.

Systematic errors incurred in the flux calibration can be quantified by comparing spectrophotometric colors for the data to published photometry. We examined $J-H, H-K$, and $J-K$ colors on the Mauna Kea Observatory system (MKO; Simons \& Tokunaga 2002; Tokunaga et al. 2002) using photometry from Geballe et al. (2001), Leggett et al. (2002), and Knapp et al. (2004); and $J-H, H-K_{s}$, and $J-K_{s}$ colors from 2 MASS (Table 1). Spectrophotometric colors were determined by integrating the appropriate filter profile (combined with telescope and instrumental optical response curves for 2MASS photometry; see $\oint$ III.1.b.i in Cutri et al. 2003) over the near-infrared spectra of each T dwarf and that of the A0 V star Vega (Bergeron et al. 1995; see also Stephens \& Leggett 2004). We found no systematic differences for any of the photometric and spectrophotometric colors on both systems, and typical deviations were 5\% or less for the more accurate MKO photometry. For those few sources with color offsets significantly greater than their photometric uncertainties (2MASS 0243-2453, 2MASS 0727+1710, and SDSS $1758+4633$ have $3 \sigma$ deviations in MKO $J-K$ ), differences were at most $15 \%$. Hence, we conclude that the $10 \%$ uncertainties adopted for the color ratios adequately compensate for uncertainties in the flux calibration.

\subsubsection{Spectral Noise}

Molecular band ratios are generally insensitive to color errors in the overall spectrum, but deep absorption bands can be affected by spectral noise. This is manifested by variations in the measured flux at the bottom of the bands, where the signal-tonoise ratio $(\mathrm{S} / \mathrm{N})$ is minimal. To explore the impact of this effect, we performed a Monte Carlo experiment, measuring the $\mathrm{H}_{2} \mathrm{O}-J$ ratio on the combined spectrum of Gliese 570D plus a Gaussian noise component scaled to $\mathrm{S} / \mathrm{N}=10-200$ at the $J$-band peak. A total of 10,000 trials were run over a uniform range of $\mathrm{S} / \mathrm{N}$. We found no systematic deviations in the $\mathrm{H}_{2} \mathrm{O}-J$ ratios for $S / N \gtrsim 10$, but scatter among the values increased in the noisier spectra, approaching $10 \%$ for $\mathrm{S} / \mathrm{N}=40$. All of our spectra have $\mathrm{S} / \mathrm{N} \gtrsim 50$ at the $J$-band peak, with the exception of SDSS $1110+0116$, which has $\mathrm{S} / \mathrm{N} \approx 20$. Hence, we find that our adopted $10 \%$ uncertainties for the measured $\mathrm{H}_{2} \mathrm{O}$ ratios incorporate noise effects sufficiently, although derived values for SDSS 1110+0116 may be more uncertain.

\subsubsection{Choice of Spectral Ratios}

The use of the $\mathrm{H}_{2} \mathrm{O}-J$ and $K / H$ ratios in our analysis above was justified by the sensitivity of these ratios to $T_{\text {eff }}$ and $g$ variations in the models, the magnitude of the calibration correction 

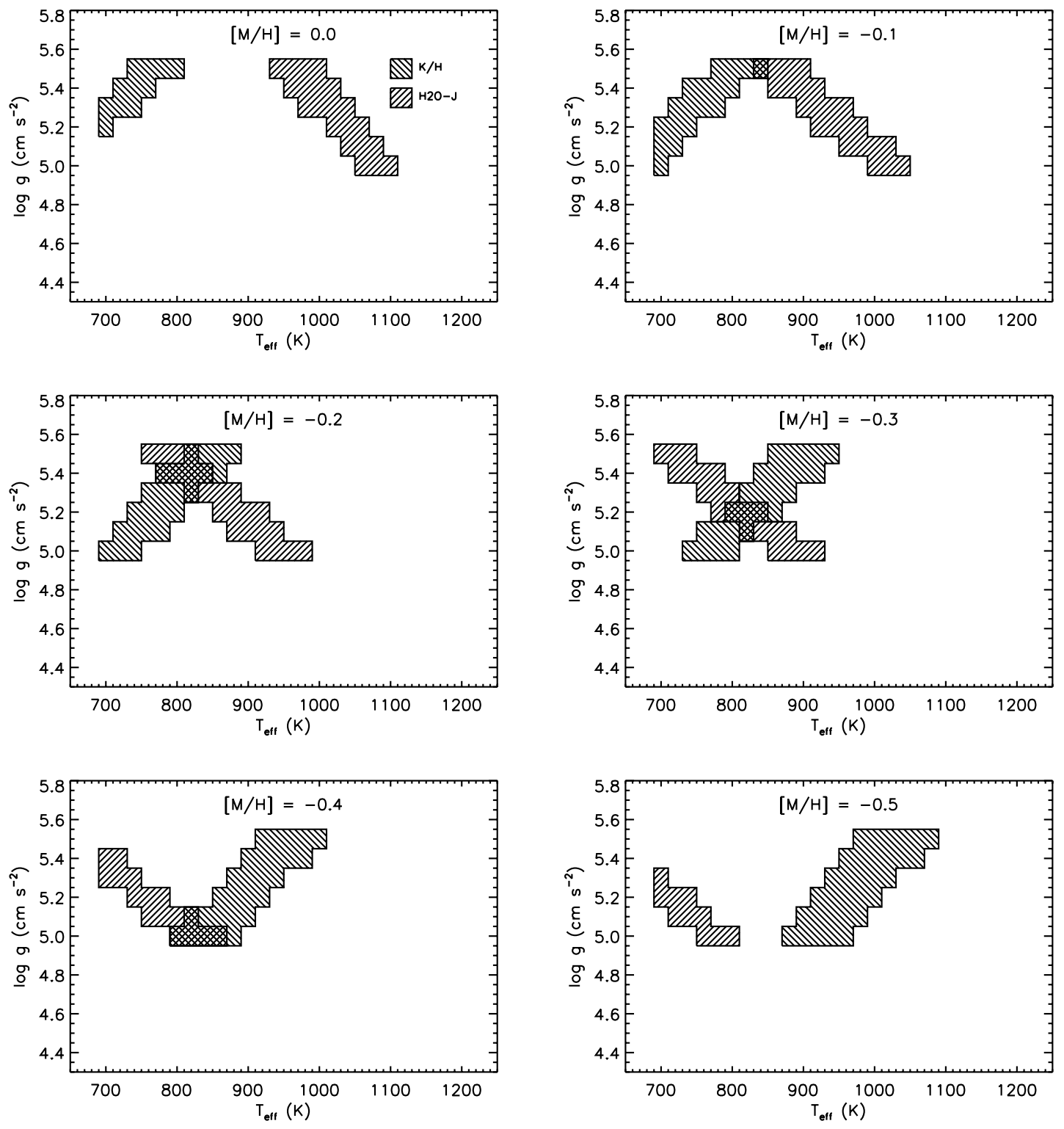

FIG. 8. - The $T_{\text {eff }}$ and $\log g$ parameter fit for 2 MASS $0937+2931$ for spectral models with metallicities $-0.5 \leq[\mathrm{M} / \mathrm{H}] \leq 0$, interpolated in steps of 0.1 dex.

required, and the fidelity of the models in these spectral regions. However, we can also consider how the results change if a different set of ratios is employed. We repeated our analysis with four pairings among the $\mathrm{H}_{2} \mathrm{O}-J, \mathrm{H}_{2} \mathrm{O}-H, K / H$, and $K / J$ ratios. Again, we found no significant or systematic differences in the derived $T_{\text {eff }}$ and $\log g$ values among our sample, although parameters for individual sources differed by as much as $160 \mathrm{~K}$ and 0.6 dex, respectively. Typical deviations were of order $65 \mathrm{~K}$ in $T_{\text {eff }}$ and $0.15 \mathrm{dex}$ in $\log g$, which we adopt as estimates of systematic uncertainty.

\subsubsection{Calibration of the Spectral Ratios}

The calibration of the spectral ratios hinges largely on the assumed physical properties for our calibrator source Gliese 570D. But how sensitive are the results to these adopted parameters? By varying the assumed $T_{\text {eff }}$ and $\log g$ for Gliese $570 \mathrm{D}$ by $\pm 25 \mathrm{~K}$ and $\pm 0.1 \mathrm{dex}$, respectively, consistent with the range of values found by Geballe et al. (2001), we found mean offsets of $\pm 35 \mathrm{~K}$ and \pm 0.1 dex in the derived parameters for the field sources. These offsets were independent; changing the adopted $T_{\text {eff }}$ of
Gliese 570D had no impact on the derived surface gravities, and vice versa. Hence, we estimate that additional systematic uncertainties of $35 \mathrm{~K}$ and $0.1 \mathrm{dex}$ in $T_{\text {eff }}$ and $\log g$, respectively, accommodate uncertainties in the physical parameters of our comparison source.

\subsubsection{Choice of Spectral Models}

Many of the potential systematic effects involving the choice and calibration of the spectral ratios would be eliminated if the models accurately reproduced the observed spectra. As limitations in the opacities prevent this, we must also consider how dependent our results are on the choice of spectral models used. We therefore repeated our analysis using the COND models of Allard et al. (2001). Calibration of the spectral ratios was performed in the same manner as with the Tucson models, yielding somewhat different correction values. While deviations typically of order $50 \mathrm{~K}$ and 0.1 dex were found when comparing derived $T_{\text {eff }}$ and $\log g$ between the model sets, these deviations were not systematic. Hence, we conclude that the choice of spectral model does not systematically change our results. 
In summary, we find no systematic deviations in our method that would lead to skewed estimates of $T_{\text {eff }}$ and $\log g$, although systematic uncertainties of order 50-100 K and 0.1-0.25 dex may be present.

\section{MASS, RADIUS, AND AGE ESTIMATES}

\subsection{Evolutionary Models}

According to brown dwarf evolutionary theory, the temperature and surface gravity of a brown dwarf at a given time is directly related to its mass and age, assuming a given composition. ${ }^{3}$ This implies that if $T_{\text {eff }}$ and $g$ are known, estimates for the latter, more fundamental physical properties could be derived on an individual basis. This is demonstrated graphically in Figure 9, which compares the derived $T_{\text {eff }}$ and $\log g$ values for 14 of our sources (excluding 2MASS 0939-2448 and 2MASS 1114-2618) to solar metallicity evolutionary models from Burrows et al. (1997). Table 5 lists the range of masses and ages, and the corresponding radii, derived from this comparison. Our sample appears to span a broad range of masses $\left(0.02-0.07 M_{\odot}\right)$ and ages $(<1-10 \mathrm{Gyr})$, consistent with a random sample drawn from the local Galactic environment. At late ages, brown dwarf radii are fairly constant, so mass and surface gravity are almost directly related. Hence, our lowest (highest) surface gravity objects are also the least (most) massive and youngest (oldest). Note in particular the placement of 2MASS $0937+2931$, which appears to be the most massive and oldest in the sample. On the other hand, our analysis suggests that the low surface gravity T dwarfs SDSS $1758+4633$ and 2MASS 2228-4310 may have masses less than $0.03 M_{\odot}$ and ages less than $1 \mathrm{Gyr}$.

How reliable are these estimates? An independent check of the derived ages can be made by examining the kinematics of our sample. Three-dimensional space velocities have not yet been measured for the $\mathrm{T}$ dwarfs examined here; a rough analysis can be made, however, by examining their tangential velocities, $V_{\tan }$. Of the 14 sources in our sample with age estimates, 13 have proper motion measurements and 8 have parallax measurements. For 2MASS 0034+0523, 2MASS 0050-3322, 2MASS 1231+0847, and 2MASS 2228-4310 we adopted spectrophotometric distance estimates from Burgasser et al. (2003c) and Tinney et al. (2005); a distance for SDSS 1110+0116 was estimated using absolute MKO $M_{J}$ and $M_{K}$ magnitude/spectral type relations from Golimowski et al. (2004). Dividing the $13 \mathrm{~T}$ dwarfs into those with mean estimated ages less than $2 \mathrm{Gyr}$ (young) and older than $2 \mathrm{Gyr}$ (old), we computed the mean $\left(\left\langle V_{\tan }\right\rangle\right)$ and standard deviation $\left(\sigma_{V_{\tan }}\right)$ for each age group. For the young sources, $\left\langle V_{\tan }\right\rangle=38 \mathrm{~km} \mathrm{~s}^{-1}$ and $\sigma_{V_{\text {tan }}}=20 \mathrm{~km} \mathrm{~s}^{-1}$, while for the old sources $\left\langle V_{\tan }\right\rangle=51 \mathrm{~km} \mathrm{~s}^{-1}$ and $\sigma_{V_{\text {tan }}}=31 \mathrm{~km} \mathrm{~s}^{-1}$. The larger mean motion and greater scatter in velocities for the latter group is consistent with an older mean age. However, a rigorous examination of the three-dimensional velocities is required before a conclusive assessment can be made.

\subsection{Independent Mass and Radius Estimates}

The masses and ages of the objects in our sample, as derived from the evolutionary models, appear to be reasonable and consistent with their overall properties. However, these values must be considered with caution, as they are susceptible to systematic errors in both the atmosphere and interior models. Disagreements in mass and radius estimates between these two types of

\footnotetext{
${ }^{3}$ We ignore for this discussion metallicity effects in the evolution of a brown dwarf, in addition to other variations related to rotation, magnetic activity, accretion, or binary interaction that can also modulate the observed parameters and evolution of a brown dwarf.
}

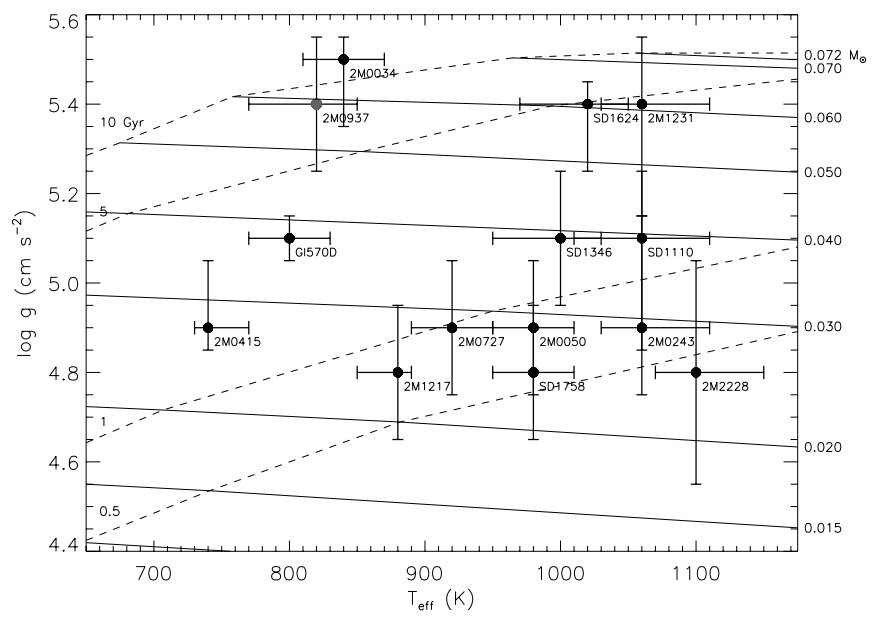

FIG. 9.-Best-fit $T_{\text {eff }}$ and $\log g$ values derived for the $\mathrm{T}$ dwarfs, as compared to evolutionary models from Burrows et al. (1997). Isochrones are indicated by dashed lines $(10,5,1$, and $0.5 \mathrm{Gyr}$, from top to bottom), while constant mass values (labeled; in solar masses) are denoted by solid lines. Error bars on the data points are based on the breadth of the $T_{\text {eff }}, \log g$ space spanned by the intersecting regions in Figs. 7 and 8 (plus an additional $10 \mathrm{~K}$ and 0.05 dex uncertainty in $T_{\text {eff }}$ and $\log g$ for sampling uncertainty); possible systematic errors of $50-100 \mathrm{~K}$ in $T_{\text {eff }}$ and $0.1-$ $0.25 \mathrm{dex}$ in $\log g$ are not included. The gray circle denotes parameters for 2MASS $0937+2931$, assuming $[\mathrm{M} / \mathrm{H}]=-0.2($ see $\S$ 4.2.2).

models have been suggested in a few young systems (Mohanty et al. 2004b; Close et al. 2005; however, see Luhman et al. 2005), and such systematic deviations may be present at late ages as well. Fortunately, masses and radii can be determined independently of the evolutionary models for those brown dwarfs with bolometric luminosity measurements.

The surface gravity of a solid body, $g=G M / R^{2}$, is applicable for brown dwarf photospheres, since the vertical scale-height of this region (a few kilometers; Griffith \& Yelle 1999) is insignificant compared to the radius of the brown dwarf itself $\left(\sim 0.1 R_{\odot} \equiv 6.95 \times 10^{4} \mathrm{~km}\right)$. Combining this with the definition of $T_{\text {eff }}, L=4 \pi R^{2} \sigma T_{\text {eff }}^{4}$, yields

$$
\begin{aligned}
M & =\frac{L g}{4 \pi G \sigma T_{\text {eff }}^{4}} \\
& =0.0408\left(\frac{L}{10^{-5} L_{\odot}}\right)\left(\frac{g}{10^{5} \mathrm{~cm} \mathrm{~s}^{-2}}\right)\left(\frac{T_{\text {eff }}}{1000 \mathrm{~K}}\right)^{-4} M_{\odot}
\end{aligned}
$$

and

$$
R=\left(\frac{L}{4 \pi \sigma T_{\mathrm{eff}}^{4}}\right)^{1 / 2}=0.106\left(\frac{L}{10^{-5} L_{\odot}}\right)^{1 / 2}\left(\frac{T_{\mathrm{eff}}}{1000 \mathrm{~K}}\right)^{-2} R_{\odot} .
$$

These equations rely only on the $T_{\text {eff }}$ and $g$ values obtained from the spectral models and the measured luminosities and not on any evolutionary model (cf. Mohanty et al. 2004b)

Luminosities for field brown dwarfs have been compiled by a number of studies (Geballe et al. 2001; Dahn et al. 2002; Nakajima et al. 2004; Cushing et al. 2005); here we focus on the results of Golimowski et al. (2004) and Vrba et al. (2004). Seven of the $\mathrm{T}$ dwarfs in our sample have luminosity determinations from these studies; Golimowski et al. also adopted the $T_{\text {eff }}$ and $\log g$ determinations of Gliese 570D from Geballe et al. (2001). The corresponding masses and radii derived from equations (6) and (7) and listed in Table 5 generally agree with those derived from the evolutionary models. Figure 10 shows a comparison of 
TABLE 5

Estimated Masses, Radil, and Ages for T Dwarfs

\begin{tabular}{|c|c|c|c|c|c|c|c|c|c|c|}
\hline \multirow[b]{2}{*}{$\begin{array}{l}\text { SourCE } \\
\text { (1) }\end{array}$} & \multirow[b]{2}{*}{$\begin{array}{c}\text { Spectral TyPe } \\
\text { (2) }\end{array}$} & \multicolumn{3}{|c|}{ Evolutionary Models $^{\mathrm{a}}$} & \multicolumn{3}{|c|}{ GolimowsKi ET AL. (2004) LuMiNosities } & \multicolumn{3}{|c|}{ VRBA ET AL. (2004) LUMINOSITIES } \\
\hline & & $\begin{array}{c}M \\
\left(M_{\odot}\right) \\
(3)\end{array}$ & $\begin{array}{c}R \\
\left(R_{\odot}\right) \\
(4)\end{array}$ & $\begin{array}{l}\text { Age } \\
\text { (Gyr) } \\
(5)\end{array}$ & $\begin{array}{c}\log L_{\text {bol }} \\
\left(L_{\odot}\right) \\
(6)\end{array}$ & $\begin{array}{c}M \\
\left(M_{\odot}\right) \\
(7)\end{array}$ & $\begin{array}{c}R \\
\left(R_{\odot}\right) \\
(8)\end{array}$ & $\begin{array}{c}\log L_{\text {bol }} \\
\left(L_{\odot}\right) \\
(9)\end{array}$ & $\begin{array}{c}M \\
\left(M_{\odot}\right) \\
(10)\end{array}$ & $\begin{array}{c}R \\
\left(R_{\odot}\right) \\
(11)\end{array}$ \\
\hline 2MASS $0034+0523 \ldots \ldots \ldots$ & $\mathrm{T} 6.5$ & $0.039-0.055$ & $0.081-0.090$ & $3.4-6.9$ & $\ldots$ & $\ldots$ & $\ldots$ & $\ldots$ & $\ldots$ & $\ldots$ \\
\hline 2MASS $0050-3322 \ldots \ldots \ldots \ldots$ & $\mathrm{T} 7$ & $0.022-0.043$ & $0.090-0.104$ & $0.5-2.5$ & $\ldots$ & $\ldots$ & $\ldots$ & $\ldots$ & $\ldots$ & $\ldots$ \\
\hline 2MASS $0243-2453 \ldots \ldots \ldots \ldots$ & $\mathrm{T} 6$ & $0.024-0.041$ & $0.092-0.106$ & $0.4-1.7$ & $-5.08 \pm 0.06$ & $0.021 \pm 0.002$ & $0.086 \pm 0.005$ & $-5.03 \pm 0.10$ & $0.023 \pm 0.003$ & $0.091 \pm 0.005$ \\
\hline 2MASS $0415-0935 \ldots \ldots \ldots \ldots \ldots$ & $\mathrm{T} 8$ & $0.022-0.044$ & $0.085-0.101$ & $1.0-4.9$ & $-5.73 \pm 0.05$ & $0.020 \pm 0.001$ & $0.083 \pm 0.002$ & $-5.18 \pm 0.10$ & $0.028 \pm 0.002$ & $0.099 \pm 0.003$ \\
\hline 2MASS $0727+1710 \ldots \ldots \ldots \ldots \ldots$ & $\mathrm{T} 7$ & $0.022-0.035$ & $0.093-0.104$ & $0.5-2.2$ & $-5.35 \pm 0.05$ & $0.020 \pm 0.002$ & $0.083 \pm 0.003$ & $-5.26 \pm 0.10$ & $0.024 \pm 0.002$ & $0.092 \pm 0.004$ \\
\hline 2MASS $0937+2931 \ldots \ldots \ldots \ldots \ldots$ & T6p & $0.047-0.063$ & $0.078-0.084$ & $5.5-10$ & $-5.28 \pm 0.05$ & $0.118 \pm 0.018$ & $0.114 \pm 0.008$ & $-5.57 \pm 0.08$ & $0.061 \pm 0.009$ & $0.081 \pm 0.006$ \\
\hline SDSS $1110+0116 \ldots \ldots \ldots \ldots \ldots$ & $\mathrm{T} 5.5$ & $0.028-0.050$ & $0.087-0.101$ & $0.5-3.4$ & $\ldots$ & & $\ldots$ & $\ldots$ & $\ldots$ & $\ldots$ \\
\hline 2MASS $1217-0311 \ldots \ldots \ldots \ldots$ & $\mathrm{T} 7.5$ & $0.022-0.038$ & $0.091-0.103$ & $0.7-2.9$ & $-5.32 \pm 0.05$ & $0.020 \pm 0.001$ & $0.094 \pm 0.002$ & $-5.30 \pm 0.09$ & $0.021 \pm 0.001$ & $0.096 \pm 0.002$ \\
\hline 2MASS $1231+0847 \ldots \ldots \ldots \ldots \ldots$ & $\mathrm{T} 5.5$ & $0.038-0.071$ & $0.078-0.093$ & $1.6-9.1$ & $\ldots$ & $\ldots$ & $\ldots$ & $\ldots$ & $\ldots$ & $\ldots$ \\
\hline SDSS $1346-0031 \ldots \ldots \ldots \ldots \ldots \ldots$ & T6.5 & $0.031-0.056$ & $0.082-0.097$ & $1.0-4.9$ & $-5.00 \pm 0.06$ & $0.051 \pm 0.006$ & $0.106 \pm 0.006$ & $-5.18 \pm 0.12$ & $0.049 \pm 0.006$ & $0.103 \pm 0.006$ \\
\hline 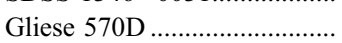 & $\mathrm{T} 7.5$ & $0.041-0.043$ & $0.087-0.089$ & $3.7-4.5$ & $-5.53 \pm 0.05^{\mathrm{b}}$ & $0.037 \pm 0.003$ & $0.090 \pm 0.004$ & $\ldots$ & $\ldots$ & $\ldots$ \\
\hline 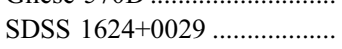 & $\mathrm{T} 6$ & $0.054-0.060$ & $0.080-0.084$ & $4.3-5.8$ & $-5.16 \pm 0.05$ & $0.065 \pm 0.007$ & $0.084 \pm 0.005$ & $-5.11 \pm 0.08$ & $0.073 \pm 0.009$ & $0.089 \pm 0.005$ \\
\hline SDSS $1758+4633 \ldots \ldots$ & $\mathrm{T} 6.5$ & $0.019-0.030$ & $0.097-0.111$ & $0.3-0.9$ & $\ldots$ & $\ldots$ & $\ldots$ & $\ldots$ & $\ldots$ & $\ldots$ \\
\hline 2MASS $2228-4310 \ldots \ldots \ldots \ldots \ldots$ & T6 & $0.018-0.034$ & $0.096-0.115$ & $0.2-0.9$ & $\ldots$ & & & $\ldots$ & $\ldots$ & $\ldots$ \\
\hline
\end{tabular}

a Estimates derived from the solar metallicity evolutionary models of Burrows et al. (1997) and $T_{\text {eff }}$ and $\log g$ determinations from Table 4.

${ }^{b} L_{\text {bol }}$ from Geballe et al. (2001) 


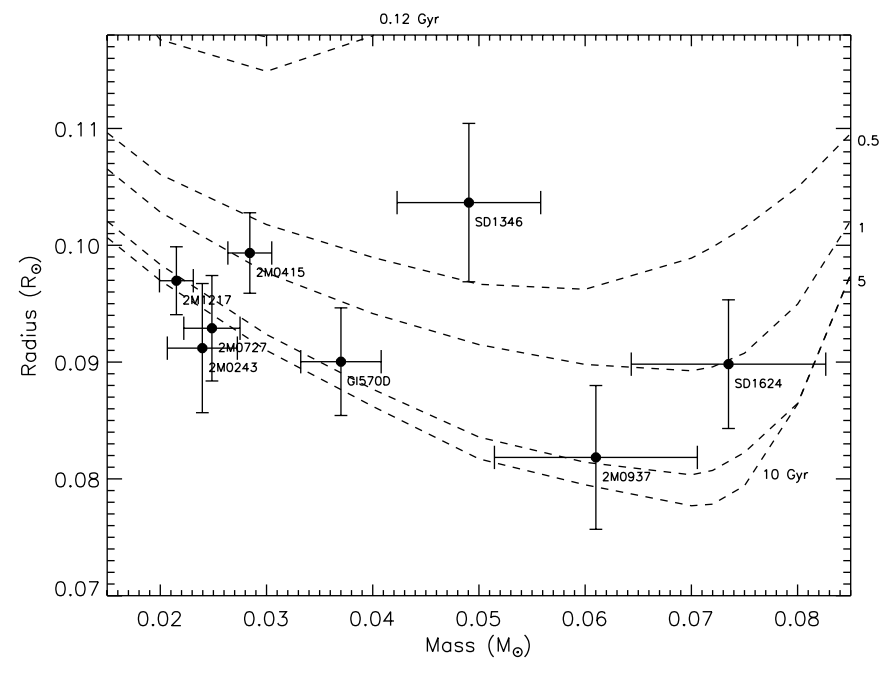

FIG. 10.-Masses and radii derived for $\mathrm{T}$ dwarfs with luminosity determinations from Vrba et al. (2004), using eqs. (6) and (7). Isochrones (ages in Gyr, as labeled) from the evolutionary models of Burrows et al. (1997) are indicated by dashed lines.

mass and radius values derived from the Vrba et al. luminosities to the Burrows et al. (1997) theoretical isochrones. With the exception of SDSS $1346-0031,{ }^{4}$ derived values lie between the 1 and 10 Gyr isochrones, as expected for a Galactic disk sample. More importantly, features in the theoretical brown dwarf massradius relationship are reproduced, including the radius minimum of $0.08 R_{\odot}$ for larger masses and the trend toward larger radii for lower mass brown dwarfs (Burrows et al. 1997; Chabrier \& Baraffe 2000). These agreements are promising and suggest that brown dwarf evolutionary tracks are robust at late ages.

Are these values truly independent of the evolutionary models? Not entirely, since the $T_{\text {eff }}$ and $\log g$ determinations hinge on the adopted values for Gliese 570D, which themselves are partly dependent on the evolutionary models. $T_{\text {eff }}$ was derived for this source by Geballe et al. (2001) using the integrated observed flux (over $0.83-2.52 \mu \mathrm{m}$ ), a bolometric correction determined from spectral models (but consistent with more recent empirical determinations; Golimowski et al. 2004), the measured parallax of the Gliese 570 system, and a radius adopted from evolutionary models. In the last case, the radii of brown dwarfs at the age of Gliese $570 \mathrm{D}$ are predicted to be roughly constant, varying by less than $20 \%$ for masses of $0.02-0.07 M_{\odot}$; are determined by well-understood interior physics; and have been empirically tested at the low-mass end with transiting extrasolar planets (e.g., Burrows et al. 2004). Hence, the $T_{\text {eff }}$ for Gliese 570D can be considered to be empirically robust. Its surface gravity, on the other hand, was derived solely from evolutionary theory. However, given the largely constant radii of old brown dwarfs $\left(R \approx 0.1 R_{\odot}\right)$ and assuming a mass in the brown dwarf range $\left(0.02-0.07 M_{\odot}\right)$ yields $\log g=4.7-5.3 \mathrm{~cm} \mathrm{~s}^{-2}$, consistent with the adopted modeldependent value. Hence, the adopted $T_{\text {eff }}$ and $\log g$ for Gliese $570 \mathrm{D}$ are only weakly tied to evolutionary models, so that the derived parameters for other $\mathrm{T}$ dwarfs can provide, at minimum, semiempirical tests of these models.

Two of the objects in our sample, 2MASS 0415-0935 and 2MASS 0937+2931, are worth additional comment, as their luminosities from Golimowski et al. (2004) and Vrba et al. (2004) are significantly discrepant. As a result, equations (6) and (7) yield

\footnotetext{
4 The large radius derived for SDSS $1346-0031$ could arise if the source is an unresolved binary, although systematic effects cannot be ruled out.
}

very different masses and radii for these sources. Golimowski et al. determined luminosities for individual brown dwarfs by integrating the total measured flux over $0.8-5.0 \mu \mathrm{m}$ (for those sources with measured $M$-band photometry), assuming a Rayleigh-Jeans tail for longer wavelengths (with corrections for molecular absorption between 4 and $15 \mu \mathrm{m}$ ) and using measured parallaxes. Vrba et al. applied a bolometric correction as a function of spectral type (computed by Golimowski et al.) to absolute $K$-band magnitudes. For 2MASS 0415-0935, the slight differences between these methods yield a lower luminosity from Golimowski et al. (by a factor of 3.5), resulting in a similar mass but a much smaller radius $\left(0.083 \pm 0.003 R_{\odot}\right)$ than that derived from the Vrba et al. measure $\left(0.092 \pm 0.004 R_{\odot}\right)$. The former estimate is outside of the Burrows et al. (1997) model parameter space. Similarly, Golimowski et al. deduced a higher luminosity for 2MASS $0937+$ 2931 than did Vrba et al., and the corresponding mass $(0.118 \pm$ $\left.0.018 M_{\odot}\right)$ and radius $\left(0.114 \pm 0.008 R_{\odot}\right)$ are well outside of the parameter space encompassed by the evolutionary models. In both cases, the Vrba et al. luminosities yield values consistent with the models. This is intriguing, since both 2MASS 0415-0935 and 2MASS $0937+2931$ have measured $M$-band photometry, and the corresponding luminosities from Golimowski et al. are expected to be more accurate. These deviations may indicate systematic errors in the luminosity determinations of either Golimowski et al. or Vrba et al., or in our $T_{\text {eff }}$ and surface gravity estimates. This is not entirely unexpected for the apparently metal-poor $\mathrm{T}$ dwarf 2MASS 0937+2931. However, in order to assess the origin of these deviations, and whether they actually indicate problems in the evolutionary models, the number and quality of luminosity measurements for low-temperature $\mathrm{T}$ dwarfs must clearly be improved.

\section{DISCUSSION}

\subsection{The Temperature Scale of Late-type T Dwarfs}

Disentangling the parameters $T_{\text {eff }}$ and $g$ for $\mathrm{T}$ dwarf spectra enables a more refined examination of the $T_{\text {eff }} /$ spectral type relation for these objects, a useful function for constraining atmospheric properties as well as distance estimation. Typically, these relations are tied to luminosity measurements and an assumed radius or range of radii for sources with unknown ages (Dahn et al. 2002; Tinney et al. 2003; Golimowski et al. 2004; Vrba et al. 2004). Studies have shown that late-type T dwarfs with identical spectral types can exhibit significant differences in their estimated $T_{\text {eff }}$ values. We formally recognize this as the additional influence of surface gravity.

Figure 11 compares the derived $T_{\text {eff }}$ values for the sources in our sample to their spectral types. Objects with low and moderate surface gravities, $\log g \leq 5.1$, exhibit a tight trend of decreasing $T_{\text {eff }}$ with increasing spectral type, largely consistent with the Golimowski et al. (2004) $T_{\text {eff }} /$ spectral type relation. Higher surface gravity objects, in particular 2MASS $0034+0523$ and 2MASS $0937+2931$, have $T_{\text {eff }}$ values that are $150-250 \mathrm{~K}$ cooler for their spectral types. This behavior can be understood by the interplay between $T_{\text {eff }}$ and $g$ on the major $\mathrm{H}_{2} \mathrm{O}$ bands, the depths of which determine in part $\mathrm{T}$ spectral types. $\mathrm{T}$ dwarfs with higher surface gravities have weaker $\mathrm{H}_{2} \mathrm{O}$ bands and hence earlier spectral types at a given $T_{\text {eff }}$; consequently, they would appear to have lower $T_{\text {eff }}$ values for a given spectral type. This gravity trend is also present when comparing luminosities, as the oldest, most massive brown dwarfs (which have the highest surface gravities) can have radii that are $10 \%-15 \%$ smaller than $1-3$ Gyr brown dwarfs (Burrows et al. 1997). Coupled with 10\%-20\% lower $T_{\text {eff }}$ values, old brown dwarfs can be up to 3 times fainter than their younger field counterparts. This is precisely the deviation 


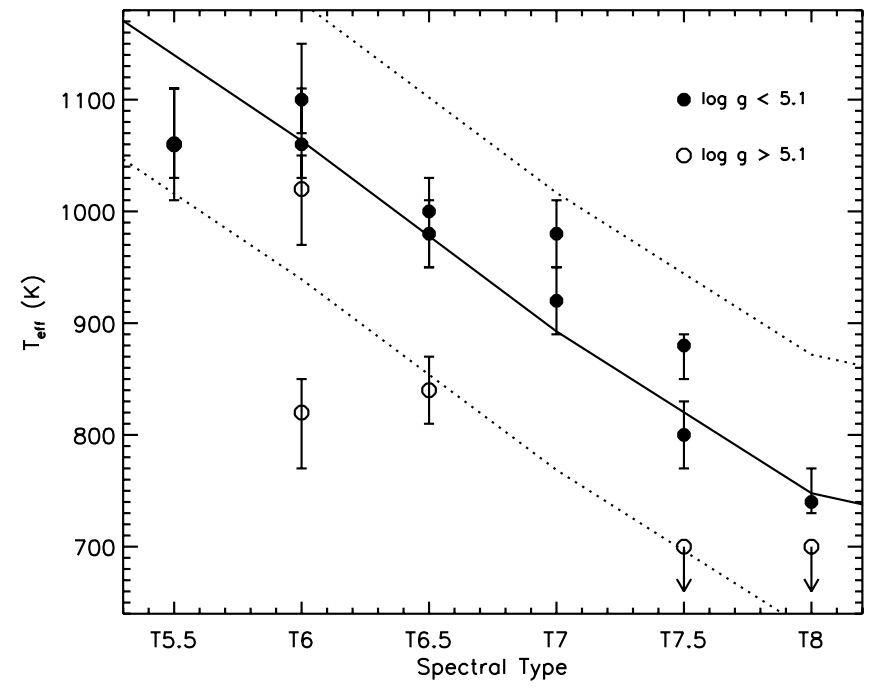

FIG. 11.-Derived $T_{\text {eff }}$ values for objects in our sample as a function of nearinfrared spectral type. Sources have been segregated into those with low and moderate surface gravities ( $\log g \leq 5.1$; filled circles) and those with high surface gravities ( $\log g>5.1$; open circles). The low and moderate surface gravity objects form a tighter trend than the full sample, as traced by the Golimowski et al. (2004) $T_{\text {eff }} /$ spectral type relation (solid line; dashed lines delineate $\pm 124 \mathrm{~K}$ uncertainty in the relation); higher surface gravity objects tend to have lower $T_{\text {eff }}$ values at a given spectral type.

Vrba et al. (2004) found in the luminosity of 2MASS 0937+2931 as compared to other T6 dwarfs (Golimowski et al. 2004 found a somewhat smaller deviation). Hence, luminosity and/or $T_{\text {eff }}$ measurements for a consistently classified sample could provide a means of segregating young and old systems.

\subsection{Improving Temperature and Surface Gravity Determinations}

The method outlined here is in some sense a response to the current limitations of the spectral models. As the models improve in accuracy, direct spectral comparisons should eventually be sufficient to determine the physical parameters offield brown dwarfs. On the other hand, our spectral index comparison method could also be improved by using additional empirical calibrators such as Gliese 570D. Additional calibrators would enable higher order corrections to the model ratios, reducing systematic effects. Such empirical constraints can be taken one step further: a sufficiently sampled parameter space of calibrator sources could enable the determination of $T_{\mathrm{eff}}$ and $g$ values independent of the spectral models. To do this, several more companion brown dwarfs with independent age and metallicity determinations, and/or binary (particularly eclipsing) systems with measured orbital parameters, are required. While Gliese 570D is currently the only such calibrator source in the late-T dwarf regime, three closely separated late-type $\mathrm{T}$ dwarf binaries have been identified for which mass measurements are feasible (Burgasser et al. 2003b; A. J. Burgasser et al. 2006, in preparation); and one T dwarf binary, $\epsilon$ Indi Bab (Scholz et al. 2003; McCaughrean et al. 2004) is also a companion to a nearby $0.8-2$ Gyr K5 V star. There are also several ongoing searches for wide brown dwarf companions to nearby and young stars (e.g., Chauvin et al. 2004, 2005; Neuhäuser et al. 2005). Identification of several such calibration stars would provide an empirical ladder for determining the physical properties of field brown dwarfs, as well as a critical test for both spectral and evolutionary models.

Can we also extend this technique to earlier spectral types: e.g., L dwarfs and early-type T dwarfs? In these regimes, spectral energy distributions are strongly affected by photospheric condensates, giving rise to what many consider to be a fourth "dust" parameter (e.g., $f_{\text {sed }}$ in Ackerman \& Marley [2001] and Marley et al. [2002]; $T_{\mathrm{cr}}$ in Tsuji [2005]; and $a_{0}$ in Burrows et al. [2005]), which may vary with the photospheric gas properties or other secondary effects, such as rotation. In principle, the method outlined here could be extended into the L and early-T dwarf regime by incorporating this fourth parameter, employing suitable dust cloud models and enlarging the sample of empirical calibration stars. In practice, this approach may prove more difficult, as brown dwarf cloud formation remains a poorly understood process (e.g., Helling et al. 2004). Nevertheless, $T_{\text {eff }}, g$, and dust-content determinations would be particularly useful for understanding the rapid dust depletion that occurs at the $\mathrm{L} / \mathrm{T}$ transition (Dahn et al. 2002; Burgasser et al. 2002a; Knapp et al. 2004).

\subsection{Applications of the Method}

The ability to determine masses and ages for individual brown dwarfs is clearly a boon to population studies of these objects, particularly the Galactic substellar MF and formation history. Constraints on these fundamental relations have largely been statistical in nature because of the difficulty in determining masses and ages for field sources (Reid et al. 1999; Chabrier 2003; Burgasser 2004; Allen et al. 2005). Using the method described here, both distributions can be built up directly from individual sources in a sample, assuming that careful consideration is made of selection effects. While current samples of late-type T dwarfs are too small for a robust analysis of this kind (Burgasser et al. 2002b; Knapp et al. 2004), searches for cold brown dwarfs from wide and deep near-infrared surveys such as the United Kingdom Infrared Telescope (UKIRT) Deep Sky Survey ${ }^{5}$ (Warren 2002) may make MF and age distribution measurements feasible in the near future.

In the more immediate term, $T_{\text {eff }}$ and $g$ measurements and corresponding mass and age estimates are useful for identifying and characterizing young, low-mass objects in young star-forming regions. According to current evolutionary models, T dwarfs with ages $\lesssim 10$ Myr can have masses of only a few Jupiter masses $\left(M_{\text {Jup }}\right)$. Several young cluster brown dwarf candidates have been identified in recent years, including the late-type T dwarf S Orionis 70 (Zapatero Osorio et al. 2002; Martín \& Zapatero Osorio 2003). Direct comparison of spectral data to theoretical models has suggested a very low surface gravity for this source, $\log g \approx 3.5-4.0$, indicative of a young, very low mass $\left(\sim 3 M_{\text {Jup }}\right)$ brown dwarf that has not yet fully contracted. However, such direct spectral comparison has been shown to be flawed for late-T spectral types in general (Burgasser et al. 2004a). A calibrated spectral ratio comparison could provide a more robust assessment of the physical properties of this and other low-mass candidates and verify their cluster membership. Furthermore, with luminosity measurements, independent determinations of mass and age would provide semiempirical constraints on the evolutionary models from which mass estimates are currently derived. Such independent assessments are necessary to validate the existence of so-called planetary-mass brown dwarfs in these young starforming regions.

\section{SUMMARY}

We have devised a method for measuring the effective temperatures and surface gravities for the lowest luminosity T-type

\footnotetext{
5 See http://www.ukidss.org/index.html.
} 
brown dwarfs, by the comparison of calibrated spectral ratios measured on low-resolution near-infrared spectral data and theoretical models. Using this method, we have derived $T_{\text {eff }}$ and $g$ estimates for 14 T5.5-T8 field brown dwarfs and a subsolar metallicity estimate for the peculiar T dwarf 2MASS 0937+ 2931. Two other sources in our sample, 2MASS 0939-2448 and 2MASS $1114-2618$, appear to have $T_{\text {eff }} \lesssim 700 \mathrm{~K}$ and are potentially the coldest brown dwarfs currently known. We find no evidence of systematic effects in our method, and the agreement of our $T_{\text {eff }}$ determinations with prior studies suggests that our results are robust. We also find that the scatter observed in $T_{\text {eff }} /$ spectral type relations likely arises from surface gravity effects, as higher surface gravity objects have a lower $T_{\text {eff }}$ at a given spectral type. Masses, radii, and ages are derived for objects in our sample using the Burrows et al. (1997) evolutionary models, while independent mass and radius determinations are made for eight $\mathrm{T}$ dwarfs with luminosity measurements. Broad agreement between these values suggests that current brown dwarf evolutionary models are accurate at late ages, although this must be verified through improved luminosity determinations.

The comparative technique described here is a useful tool for determining the physical properties of the lowest luminosity brown dwarfs, making efficient use of low-resolution, and therefore more sensitive, spectroscopy. As such, it is a promising method for characterizing large samples arising from deep surveys, enabling a direct determination of the Galactic substellar MF and formation history. While our method remains tied to the current generation of spectral models, susceptible to persistent opacity deficiencies, the increased use offiducial calibrators will ultimately enable a wholly empirical approach, allowing critical tests of atmospheric and evolutionary theories in addition to the characterization of individual brown dwarfs in the vicinity of the Sun.

We thank our telescope operators, Dave Griep and Paul Sears, and instrument specialist John Rayner, for their support during the SpeX observations. We also acknowledge extremely helpful comments from our referee, Gibor Basri, and editor, James Liebert, which allowed us to substantially improve on our original manuscript. A. Burgasser thanks P. Hauschildt for making electronic versions of the COND models available for analysis. A. Burrows acknowledges support under NASA grant NNG 04GL22G. This publication makes use of data from 2MASS, which is a joint project of the University of Massachusetts and the Infrared Processing and Analysis Center, funded by the National Aeronautics and Space Administration and the National Science Foundation. 2MASS data were obtained from the NASA/IPAC Infrared Science Archive, which is operated by the Jet Propulsion Laboratory, California Institute of Technology, under contract with the National Aeronautics and Space Administration. The theoretical material is based on work enabled by the National Aeronautics and Space Administration through the NASA Astrobiology Institute, under cooperative agreement CAN-02-OSS02 issued through the Office of Space Science. The authors wish to recognize and acknowledge the very significant cultural role and reverence that the summit of Mauna Kea has always had within the indigenous Hawaiian community. We are most fortunate to have the opportunity to conduct observations from this sacred mountain.

\section{Facilities: IRTF(SpeX)}

Ackerman, A. S., \& Marley, M. S. 2001, ApJ, 556, 872

Allard, N. F., Allard, F., Hauschildt, P. H., Kielkopf, J. F., \& Machin, L. 2003, A\&A, 411, L473

Allard, F., Hauschildt, P. H., Alexander, D. R., Tamanai, A., \& Schweitzer, A. 2001, ApJ, 556, 357

Allen, P. R., Koerner, D. W., Reid, I. N., \& Trilling, D. E. 2005, ApJ, 625, 385

Allende Prieto, C., \& Lambert, D. L. 2000, AJ, 119, 2445

Bergeron, P., Wesemael, F., \& Beauchamp, A. 1995, PASP, 107, 1047

Borysow, A., Jørgensen, U. G., \& Zheng, C. 1997, A\&A, 324, 185

Burgasser, A. J. 2004, ApJS, 155, 191

Burgasser, A. J., Geballe, T. R., Leggett, S. K., Kirkpatrick, J. D., \& Golimowski, D. A. 2006, ApJ, 637, 1067

Burgasser, A. J., Kirkpatrick, J. D., Liebert, J., \& Burrows, A. 2003a, ApJ, 594, 510

Burgasser, A. J., Kirkpatrick, J. D., McGovern, M. R., McLean, I. S., Prato, L., \& Ried, I. N. 2004a, ApJ, 604, 827

Burgasser, A. J., Kirkpatrick, J. D., Reid, I. N., Brown, M. E., Miskey, C. L., \& Gizis, J. E. 2003b, ApJ, 586, 512

Burgasser, A. J., Marley, M. S., Ackerman, A. S., Saumon, D., Lodders, K., Dahn, C. C., Harris, H. C., \& Kirkpatrick, J. D. 2002a, ApJ, 571, L151

Burgasser, A. J., McElwain, M. W., \& Kirkpatrick, J. D. 2003c, AJ, 126, 2487

Burgasser, A. J., McElwain, M. W., Kirkpatrick, J. D., Cruz, K. L., Tinney,

C. G., \& Reid, I. N. 2004b, AJ, 127, 2856

Burgasser, A. J., et al. 1999, ApJ, 522, L65

- 2000, ApJ, 531, L57

2002b, ApJ, 564, 421 2003d, ApJ, 592, 1186

Burrows, A., Burgasser, A. J., Kirkpatrick, J. D., Liebert, J., Milsom, J. A., Sudarsky, D., \& Hubeny, I. 2002, ApJ, 573, 394

Burrows, A., Hubbard, W. B., Lunine, J. I., \& Liebert, J. 2001, Rev. Mod. Phys., 73, 719

Burrows, A., Hubeny, I., Hubbard, W. B., Sudarsky, D., \& Fortney, J. J. 2004, ApJ, 610, L53

Burrows, A., Marley, M. S., \& Sharp, C. M. 2000, ApJ, 531, 438

Burrows, A., \& Sharp, C. M. 1999, ApJ, 512, 843

Burrows, A., Sudarsky, D., \& Hubeny, I. 2006, ApJ, in press

Burrows, A., Sudarsky, D., \& Lunine, J. I. 2003, ApJ, 596, 587
Burrows, A., \& Volobuyev, M. 2003, ApJ, 583, 985

Burrows, A., et al. 1997, ApJ, 491, 856

Chabrier, G. 2003, PASP, 115, 763

Chabrier, G., \& Baraffe, I. 2000, ARA\&A, 38, 337

Chauvin, G., Lagrange, A.-M., Dumas, C., Zuckerman, B., Mouillet, D., Song, I.,

Beuzit, J.-L., \& Lowrance, P. 2004, A\&A, 425, L29

Chauvin, G., et al. 2005, A\&A, 438, L29

Close, L. M., et al. 2005, Nature, 433, 286

Cushing, M. C., Rayner, J. T., \& Vacca, W. D. 2005, ApJ, 623, 1115

Cushing, M. C., Vacca, W. D., \& Rayner, J. T. 2004, PASP, 116, 362

Cutri, R. M., et al. 2003, Explanatory Supplement to the 2MASS All Sky Data

Release (Pasadena: IPAC), http://www.ipac.caltech.edu/2mass/releases/allsky/ doc/explsup.html

Dahn, C. C., et al. 2002, AJ, 124, 1170

ESA. 1997, The Hipparcos and Tycho Catalogues (ESA SP-1200; Noordwijk:

ESA)

Fegley, B., \& Lodders, K. 1996, ApJ, 472, L37

Feltzing, S., \& Gustafsson, B. 1998, A\&AS, 129, 237

Geballe, T. R., Saumon, D., Leggett, S. K., Knapp, G. R., Marley, M. S., \&

Lodders, K. 2001, ApJ, 556, 373

Geballe, T. R., et al. 2002, ApJ, 564, 466

Golimowski, D. A., et al. 2004, AJ, 127, 3516

Gorlova, N. I., Meyer, M. R., Rieke, G. H., \& Liebert, J. 2003, ApJ, 593, 1074

Griffith, C. A., \& Yelle, R. V. 1999, ApJ, 519, L85

Helling, C., Klein, R., Woitke, P., Nowak, U., \& Sedlmayr, E. 2004, A\&A, 423, 657

Homeier, D., Hauschildt, P. H., \& Allard, F. 2003, in IAU Symp. 211, Brown

Dwarfs, ed. E. L. Martín (San Francisco: ASP), 419

Kirkpatrick, J. D. 2005, ARA\&A, 43, 195

Kirkpatrick, J. D., et al. 1999, ApJ, 519, 802

Knapp, G., et al. 2004, AJ, 127, 3553

Leggett, S. K., Golimowski, D. A., Fan, X., Geballe, T. R., \& Knapp, G. R.

2003, in The Future of Cool-Star Astrophysics, ed. A. Brown, G. M. Harper,

\& T. R. Ayres (Boulder: Univ. Colorado), 120

Leggett, S. K., et al. 2002, ApJ, 564, 452

Linsky, J. L. 1969, ApJ, 156, 989

Lodders, K. 1999, ApJ, 519, 793 
Lodders, K., \& Fegley, B. 2002, Icarus, 155, 393

Lucas, P. W., Roche, P. F., Allard, F., \& Hauschildt, P. H. 2001, MNRAS, 326, 695

Luhman, K. L., Stauffer, J. R., \& Mamajek, E. E. 2005, ApJ, 628, L69

Marley, M. S., Seager, S., Saumon, D., Lodders, K., Ackerman, A. S., Freedman, R., \& Fan, X. 2002, ApJ, 568, 335

Martín, E. L., \& Zapatero Osorio, M. R. 2003, ApJ, 593, L113

McCaughrean, M., Close, L. M., Scholz, R.-D., Lenzen, R., Biller, B., Brandner, W., Hartung, M., \& Lodieu, N. 2004, A\&A, 413, 1029

McGovern, M., Kirkpatrick, J. D., McLean, I. S., Burgasser, A. J., Prato, L., \& Lowrance, P. J. 2004, ApJ, 600, 1020

McLean, I. S., McGovern, M. R., Burgasser, A. J., Kirkpatrick, J. D., Prato, L., \& Kim, S. 2003, ApJ, 596, 561

Mohanty, S., Basri, G., Jayawardhana, R., Allard, F., Hauschildt, P., \& Ardila, D. 2004a, ApJ, 609, 854

Mohanty, S., Jayawardhana, R., \& Basri, G. 2004b, ApJ, 609, 885

Nakajima, T., Tsuji, T., \& Yanagisawa, K. 2004, ApJ, 607, 499

Neuhäuser, R., Guenther, E. W., Wuchterl, G., Mugrauer, M., Bedalov, A., \& Hauschildt, P. H. 2005, A\&A, 435, L13

Rayner, J. T., Toomey, D. W., Onaka, P. M., Denault, A. J., Stahlberger, W. E., Vacca, W. D., Cushing, M. C., \& Wang, S. 2003, PASP, 115, 362

Reid, I. N., Kirkpatrick, J. D., Gizis, J. E., Dahn, C. C., Monet, D. G., Williams, R. J., Liebert, J., \& Burgasser, A. J. 2000, AJ, 119, 369

Reid, I. N., et al. 1999, ApJ, 521, 613

Saumon, D., Bergeron, P., Lunine, J. I., Hubbard, W. B., \& Burrows, A. 1994, ApJ, 424, 333
Saumon, D., Geballe, T. R., Leggett, S. K., Marley, M. S., Freedman, R. S., Lodders, K., Fegley, B., Jr., \& Sengupta, S. K. 2000, ApJ, 541, 374

Saumon, D., Marley, M. S., Lodders, K., \& Freedman, R. S. 2003, in IAU Symp. 211, Brown Dwarfs, ed. E. L. Martín (San Francisco: ASP), 345 Scholz, R.-D., Lodieu, N., \& McCaughrean, M. J. 2004, A\&A, 428, L25

Scholz, R.-D., McCaughrean, M. J., Lodieu, N., \& Kuhlbrodt, B. 2003, A\&A, 398, L29

Simons, D. A., \& Tokunaga, A. T. 2002, PASP, 114, 169

Stephens, D. C., \& Leggett, S. K. 2004, PASP, 116, 9

Strauss, M. A., et al. 1999, ApJ, 522, L61

Thoren, P., \& Feltzing, S. 2000, A\&A, 363, 692

Tinney, C. G., Burgasser, A. J., \& Kirkpatrick, J. D. 2003, AJ, 126, 975

Tinney, C. G., Burgasser, A. J., Kirkpatrick, J. D., \& McElwain, M. W. 2005, AJ, 130, 2326

Tokunaga, A. T., Simons, D. A., \& Vacca, W. D. 2002, PASP, 114, 180

Tsuji, T. 2005, ApJ, 621, 1033

Tsuji, T., Ohnaka, K., \& Aoki, W. 1996, A\&A, 305, L1 1999, ApJ, 520, L119

Tsvetanov, Z. I., et al. 2000, ApJ, 531, L61

Vacca, W. D., Cushing, M. C., \& Rayner, J. T. 2003, PASP, 115, 389

Vrba, F. J., et al. 2004, AJ, 127, 2948

Warren, S. 2002, Proc. SPIE, 4836, 313

York, D. G., et al. 2000, AJ, 120, 1579

Zapatero Osorio, M. R., Béjar, V. J. S., Martí, E. L., Rebolo, R., Barrado y Navascuès, D., Mundt, R., Eislöffel, J., \& Caballero, J. A. 2002, ApJ, 578, 536 